\title{
HOMOGENEOUS MANIFOLDS WITH NEGATIVE CURVATURE. I
}

\author{
BY \\ ROBERT AZENCOTT AND EDWARD N. WILSON( $\left.{ }^{\mathbf{1}}\right)$
}

\begin{abstract}
This paper solves the problem of determining which Lie groups act simply transitively on a Riemannian manifold with negative curvature. The results obtained extend those of Heintze for the case of strictly negative curvature. Using results of Wolf and Heintze, it is established that every connected, simply connected, homogeneous manifold $M$ with negative curvature admits a Lie group $S$ acting simply transitively by isometries and every group with this property must be solvable. Formulas for the curvature tensor on $M$ are established and used to show that the Lie algebra of any such group $S$ must satisfy a number of structural conditions. Conversely, given a Lie algebra satisfying these conditions and any member of an easily constructed family of inner products on b, a metric deformation argument is used to obtain a modified inner product which gives rise to a left invariant Riemannian structure with negative curvature on the associated simply connected Lie group.
\end{abstract}

1. Introduction. This paper was motivated by the following problem: Which connected Lie groups admit a left invariant Riemannian metric with negative (sectional) curvature? We emphasize that throughout the paper, we understand "negative" to mean "less than or equal to zero". Since the property in question is not sensitive to groups linked by a local isomorphism, we deal primarily with simply connected groups. Results of J. A. Wolf [13] and E. Heintze [4] show that the above problem is closely linked with the classification of connected, homogeneous Riemannian manifolds with negative curvature. Indeed, if $M$ is such a manifold and if $M$ is simply connected, then $M$ is isometric to a solvable Lie group endowed with a left-invariant metric.

In this paper, we give a complete solution to our original problem by showing that a necessary and sufficient condition for a group to have the property in question is that its Lie algebra be what we call an "NC algebra". Roughly speaking, the crucial properties of an $N C$ algebra are that in addition to being solvable, 8 must contain an abelian subalgebra a complementary to the derived

Received by the editors August 8, 1974 and, in revised form, February 24, 1975.

AMS (MOS) subject classifications (1970). Primary 57E25, 22E25; Secondary 17B30, $53 \mathrm{C} 25$, 43A85.

Key words and fhrases. Simply transitive isometry groups, solvable Lie groups, negative curvature Lie algebras, almost normal operators, Lie algebra deformations.

(1) The authors of this paper were partially supported through NSF Grant No. GP-283-23A at Brandeis University. 
subalgebra $\mathfrak{n}$ of 8 and that a must contain elements $H_{0}$ such that $\alpha\left(H_{0}\right)>0$ whenever $\alpha \in a^{*}-\{0\}$ is the real part of a root of the adjoint representation of $\boldsymbol{a}$ on the complexification of $\mathfrak{n}$. In addition, there are several technical properties linking the structure of $\mathfrak{n}$ with the extent to which the adjoint action of $\mathfrak{a}$ on $\mathfrak{n}$ can deviate from a semisimple action. See Definition 6.2 for a precise description of $N C$ algebras. We wish to point out that in the announcement of our results in [1], Theoreme 3 is incorrectly stated because of the omission of the property given here in $6.2(\mathrm{v})$.

For the case of strictly negative curvature (see $\$ 7.8$ ), the problem was recently solved by E. Heintze [4]. Here one looks only at those $N C$ algebras where the derived subalgebra has codimension 1 and most of the technical difficulties inherent in the general situation are not present. We wish to thank J. A. Wolf for bringing Heintze's results to our attention.

The second part of our work, to appear elsewhere, will use the results obtained here to study in greater detail the structure of the group $I(M)$ of all isometries on a homogeneous Riemannian manifold $M$ with negative curvature and will push further towards a classification of these manifolds up to isometries. In particular, we shall give a complete group-theoretic characterization of those Lie groups isomorphic to $I_{0}(M)$ for $M$ a simply connected manifold of the above type. See $\$ 8$ for further comments on the questions to be dealt with in Part II.

We conclude this introduction with a summary of the organization of results in the present paper. A first good look (\$2) into the structure of $I(M)$ for $M$ a homogeneous manifold with negative curvature reduces the initial problem to the context of solvable groups. Hence, in $\S 3$, we fix a solvable group $S$ endowed with a left-invariant metric and compute explicitly the sectional curvature on 8 , the Lie algebra of $S$. Imposing the condition of negative curvature, the computation shows that if $H \in \mathbf{8}$ is orthogonal to $\mathfrak{n}=[8,8]$, then the operator $A=$ ad $\left.H\right|_{\mathfrak{n}}$ is "almost normal" in the sense that $(\operatorname{Re} A)^{2}+[\operatorname{Re} A, \operatorname{Im} A] \geqslant 0$, where $\operatorname{Re} A$ and $\operatorname{Im} A$ are, respectively, the symmetric and skew-symmetric parts of $A$. $\$ 4$ begins with a rather painful clarification of the structure of certain families of almost normal operators. For the case at hand, this information shows that $a$, the orthogonal complement of $\mathfrak{n}$ in $\mathbf{8}$, is abelian and provides information about root subspaces of $\mathfrak{n}$ relative to the adjoint action of $\mathfrak{a}$. More refined structural constraints on 8 are obtained in $\S 5$, where in particular it is seen that the nonzero real parts of the roots of $\mathfrak{a}$ in $\mathfrak{n}$ lie in an open half-space of the dual $\mathfrak{a}^{*}$ of a. These constraints are then collected together in $\$ 6$ to form the definition of the class of "NC algebras". Finally, in $\S 7$, we prove that when 8 is an arbitrary $N C$ algebra, any associated Lie group carries (many) left-invariant metrics with negative curvature. We first handle the case when $\mathfrak{n}=[8,8]$ is abelian by explicit diagonalization of the curvature tensor. In the general situation, we begin with 
any "admissible" metric on 8, i.e. an inner product linked to the Lie structure of 8 by a few simple constraints. By linear "deformation" of this metric, we obtain the desired metrics on 8 . The main point is that the study of linear deformations of a metric is in an appropriate sense dual to the study of the fixed metric together with linear deformations of the Lie structure on 8 tending to make $\mathfrak{n}$ "almost abelian".

2. Structure of the group of isometries.

2.1. Let $M$ be a connected Riemannian manifold and $I(M)$ the group of all isometries of $M$. Equipped with the compact open topology, $I(M)$ has a Lie group structure (Myers-Steenrod [11]). We denote by $I_{0}(M)$ the connected component of the identity in $I(M)$.

Recall that $M$ is said to be homogeneous if $I(M)$ acts transitively on $M$. In this case, $I_{0}(M)$ acts transitively on $M$ as well, the orbits of $I_{0}(M)$ being open in $M$. Moreover, a homogeneous Riemannian manifold is always complete (Kobayashi-Nomizu [9, p. 176]).

For $p \in M$, let $T_{p}(M)$ denote the tangent space of $M$ at $p,\langle\cdot, \cdot\rangle_{p}$ the Riemannian inner product on $T_{p}(M)$, and $|X|=\langle X, X\rangle_{p}^{1 / 2}$ the length of $X \in T_{p}(M)$. Let $R$ denote the Riemann curvature tensor on $M$. The sectional curvature of $M$ at $p$ along a two-dimensional subspace $V$ in $T_{p}(M)$ is given by the formula

$$
\Phi_{p}(V)=-\left\langle R_{p}(X, Y) X, Y\right\rangle_{p} /\left(|X|^{2}|Y|^{2}-\langle X, Y\rangle_{p}^{2}\right)
$$

where $X, Y$ are any two independent elements in $V$. The manifold $M$ is said to have negative curvature (respectively strictly negative curvature) at $p$ if $\Phi_{p}(V) \leqslant 0$ (respectively $\left.\Phi_{p}(V)<0\right)$ for every two-dimensional subspace $V$ in $T_{p}(M)$. If $M$ is homogeneous, negative curvature at a single point is of course equivalent to negative curvature at all points.

As shown by Wolf [13], a connected, simply connected, homogeneous Riemannian manifold with negative curvature admits a transitive solvable group of isometries. In [4, Proposition 1], Heintze proved that in the same situation, it is actually possible to find in $I(M)$ a simply transitive solvable group of isometries. Thus such manifolds can be represented as simply connected solvable Lie groups with a left-invariant metric. This justifies the following definition.

2.2. Definition. A connected, simply connected, homogeneous Riemannian manifold with negative curvature will be called a solvmanifold with negative curvature.

In Proposition 2.5, we shall improve slightly the results of Wolf and Heintze just mentioned.

On any Riemannian manifold $M$, the Cartan-Ambrose theorem asserts that the stability subgroup in $I(M)$ of any point $p \in M$ is compact (see Helgason [5, 
pp. 167-169]). If $M$ is homogeneous and has only finitely many connected components, it follows immediately that $I(M) / I_{0}(M)$ is finite. When $M$ is a complete, simply connected, Riemannian manifold with negative curvature, a result of E. Cartan [3, Appendix III] provides a converse to the Cartan-Ambrose theorem in the sense that for any compact subgroup $K$ of $I(M)$, there is a point $p \in M$ fixed by $K$. The first rigorous proof of this result was given by A. Borel in [2]. See also Helgason [5, p. 75]. The existence and conjugacy of maximal compact subgroups in any connected Lie group (and thus in any Lie group with finitely many components) is established in the work of Malcev [10, p. 176] and Iwasawa [7, p. 532]. Combining the results just cited, we see that for $M$ a solvmanifold with negative curvature, $I(M) / I_{0}(M)$ is finite, any compact subgroup of $I(M)$ fixes a point in $M$, and $K$ is a maximal compact subgroup of $I(M)$ if and only if $K$ is the stability subgroup in $I(M)$ of some point $p \in M$.

2.3. A Riemannian manifold $M$ is said to have no Euclidean factor if $M$ is not isometric to the product of a Euclidean space of strictly positive dimension and another Riemannian manifold.

By de Rham's decomposition theorem, (see Kobayashi-Nomizu [9, pp. 192 and 240]), if $M$ is a solvmanifold with negative curvature, $M$ is isometric to a product $M_{0} \times M_{+}$where $M_{0}$ is a Euclidean space and $M_{+}$a solvmanifold with negative curvature having no Euclidean factor. The factors $M_{0}$ and $M_{+}$are uniquely determined (up to isometry) by $M$ and the group $I(M)$ is canonically isomorphic to $I\left(M_{0}\right) \times I\left(M_{+}\right)$.

Since Wolf [13] showed that $I_{0}\left(M_{+}\right)$has trivial center, it follows trivially from the above decomposition that $I_{0}(M)$ always has trivial center when $M$ is a solvmanifold with negative curvature.

We now establish an elementary result.

2.4. LEMMA. Let $M$ be a solvmanifold with negative curvature and $G a$ connected subgroup of $I(M)$ acting transitively on $M$. Suppose that $K$ is a maximal compact subgroup of $G$ and $S$ a connected Lie subgroup of $G$ acting transitively on $M$. Denote by $\mathfrak{8}, \mathbf{8}$, and the Lie algebras of $G, S$ and $K$. If $\& \cap \mathfrak{l}=$ $\{0\}, S$ is simply connected, closed in $G$, and acts simply transitively on $M$.

Proof. Let $p$ be a point in $M$ such that $K$ is the stability group of $p$ in $G$. The action of $G$ on $M$ defines a continuous map $F$ from $S$ onto $M$ such that $F(s)=s \cdot p$. The stability group of $p$ in $S$ is $S \cap K$ which is therefore closed in $S$. Since its Lie algebra is $8 \cap \mathfrak{t}=\{0\}, S \cap K$ is discrete and $F$ is a covering map. But $M$ is simply connected, so $S \cap K=\{e\}$ and $S$ acts simply transitively on $M$. As is easily checked, $d F$ is everywhere nonsingular and $F$ is a diffeomorphism from $S$ onto $M$. Let $s_{n}$ be a sequence of elements in $S$ converging to $g \in G$. Then $s_{n} \cdot p$ converges to $q=g \cdot p$. But $q=s \cdot p$ for some unique 
element $s \in S$ and it follows that $s_{n}$ converges to $s$. Therefore $S$ is closed in $G$. Q.E.D.

2.5. Proposition. Let $M$ be a solvmanifold with negative curvature and $G$ any connected Lie subgroup of $I(M)$ acting transitively on $M$. Let $G=G_{1} \cdot G_{2}$ be a Levi decomposition of $G$ (i.e. $G_{2}$ is the radical of $G, G_{1}$ is a closed connected semisimple subgroup of $G$, and $G_{1} \cap G_{2}$ is discrete). Then $G_{1}$ has finite center and for each Iwasawa decomposition $G_{1}=K_{1} S_{1}$ ( $K_{1}$ maximal compact in $G_{1}$, $S_{1}$ closed solvable in $G_{1}$ ) there is a closed subgroup $S_{2}$ of $G_{2}$, normal in $G$, such that $S_{1} \cap S_{2}=\{e\}$ and $S=S_{1} \cdot S_{2}$ is a closed simply connected solvable subgroup of $G$ acting simply transitively on $M$.

Proof. As seen in 2.3, the center of $I_{0}(M)$ is trivial. Hence $I_{0}(M)$ and, a fortiori, any subgroup of $I_{0}(M)$, is isomorphic to a group of complex matrices. In particular, with the notations introduced in Proposition 2.5, $G_{1}$ has finite center.

Let $H=S_{1} G_{2}$. Since $G=H K_{1}$ and $K_{1}$, being compact, must leave some $p \in M$ fixed, we conclude that $H$ is a solvable group acting transitively on $M$.

Let $K_{2}$ be the subgroup of $H$ leaving $p$ fixed. Under the isomorphism of $G$ with a matrix group given above, we may regard $K=K_{1} K_{2}$ as a group of unitary matrices, and, by Lie's theorem, $H$ as a group of upper triangular matrices. Thus $K_{2}=K \cap H$ is abelian and $K_{2} \cap[H, H]=\{e\}$ where $[H, H]$ is the commutator subgroup of $H$. Moreover, $K_{2}$ must be a subgroup of $G_{2}$. To see this note that $S_{1} \cap G_{2}=\{e\}$ since $G_{1} \cap G_{2}$ is a subgroup of the center of $G_{1}$ and hence is in $K_{1}$. But $S_{1}$ and $G_{2}$ are closed in $G$ and $S_{1}$ normalizes $G_{2}$; it follows easily that the topology on $H=S_{1} G_{2}$ is the product topology, and $S_{1}$ is diffeomorphic to $H / G_{2}$ under the canonical map $\pi(g)=g G_{2}$. Therefore $\pi\left(K_{2}\right)$ is trivial since $S_{1}$ has no nontrivial compact subgroups.

Now let $\boldsymbol{g}_{2}, \mathfrak{l}_{2}$ be the Lie algebras of $G_{2}, K_{2}$, and $\mathfrak{n}_{2}$ the derived algebra of $\mathbf{g}_{2}$. From above, we have $\mathfrak{l}_{2} \subset g_{2}$ and $\mathfrak{l}_{2} \cap \mathfrak{n}_{2}=\{0\}$. Consider the connected abelian group $A=G_{2} / N_{2}$ where $N_{2}$ is the (closed) connected Lie subgroup of $G_{2}$ with Lie algebra $\mathfrak{n}_{2}$. The image of $K_{2}$ in $A$ lies in the maximal torus $T$ of $A$. The action of $G$ by automorphisms on $G_{2}$ defines a continuous action of $G$ by automorphisms on $A$. The automorphisms of $A$ leave $T$ invariant, and the automorphism group of a torus is discrete. Since $G$ is connected we conclude that inner automorphisms of $G$ leave $K_{2}$ fixed modulo $N_{2}$. By differentiation, the adjoint action of $G$ leaves $\mathfrak{l}_{2}+\mathfrak{n}_{2}$ invariant. Since $G_{1}$ is semisimple we may find a $G_{1}$-invariant subspace $a_{2}$ in $g_{2}$ complementary to $\mathfrak{l}_{2}+\mathfrak{n}_{2}$. Define $\boldsymbol{g}_{2}=$ $a_{2}+n_{2}$. Then $\boldsymbol{g}_{2}$ is a solvable ideal in 8 and $g_{2}$ is the vector space direct sum of $f_{2}$ and $\delta_{2}$. Hence $\delta=\delta_{1}+\delta_{2}$ is a solvable subalgebra of $\boldsymbol{g}$, complementary to $\mathrm{l}$, the Lie algebra of the maximal compact subgroup $K=K_{1} K_{2}$ of $G$. If $S$ is 
the connected Lie subgroup of $G$ with Lie algebra $8, S$ is clearly transitive on $M$ and by Lemma $2.4, S$ is closed in $G$, simply connected, and acts simply transitively on $M$. Q.E.D.

2.6. Corollary. Let $M$ be a solvmanifold with negative curvature. Any Lie subgroup of $I(M)$ acting transitively on $M$ contains a closed solvable subgroup of $I(M)$ acting simply transitively on $M$. In particular, if $S$ is any connected Lie group, and if there is on $S$ a left-invariant metric with negative curvature, $S$ must be solvable.

Proof. If a Lie subgroup $G$ of $I(M)$ is transitive on $M$ we apply Proposition 2.5 to $G$ to get the first half of the corollary. Now for $S$ as in the second half of the corollary, its simply connected covering $\widetilde{S}$ has the same property. By Lemma 2.4., $\widetilde{S}$ must be closed in $I(\widetilde{S})$ and hence $\tilde{S}$ contains a solvable subgroup acting simply transitively on the manifold $\widetilde{S}$. But the only transitive subgroup is $\widetilde{S}$ itself, so $\widetilde{S}$ and $S$ are solvable. Q.E.D.

2.7. Clearly, Proposition 2.5 implies the Wolf-Heintze result recalled above which represents solvmanifolds with negative curvature by solvable Lie groups endowed with left-invariant metrics. This representation will be used throughout most of the paper.

3. Computation of sectional curvature.

3.1. We continue with the solvable Lie group presentation of our manifolds. Let $S$ be a simply connected Lie group with Lie algebra 8 . As usual, 8 may be regarded either as the tangent space $T_{e}(S)$ or as the collection of left-invariant vector fields on $S$. Assume $S$ is endowed with a left-invariant Riemannian metric defined by an inner product $\langle\cdot, \cdot\rangle$ on $\mathbf{8}$. Let $\nabla$ be the Riemannian connection on $S$. By the invariance of this connection under isometries, $\nabla_{X} Y$ is a left-invariant vector field on $S$ whenever $X, Y$ are left-invariant vector fields. We still denote by $\nabla$ the induced mapping from 8 into 8 . The classical formula for a Riemannian connection (Helgason $[5$, p. 48]) reduces to

$$
2\left\langle\nabla_{X} Y, Z\right\rangle=\langle[X, Y], Z\rangle+\langle X,[Z, Y]\rangle+\langle Y,[Z, X]\rangle
$$

for $X, Y, Z$ in 8 . Clearly $\nabla_{X}$ is a skew-symmetric linear endomorphism on 8 for all $X \in 8$.

Classically, the curvature tensor $R$ defines for $X, Y \in \&$ a skew-symmetric linear endomorphism $R(X, Y)$ on $\&$ given by

$$
R(X, Y)=\left[\nabla_{X}, \nabla_{Y}\right]-\nabla_{[X, Y]}
$$

where $\left[\nabla_{X}, \nabla_{Y}\right]=\nabla_{X} \nabla_{Y}-\nabla_{Y} \nabla_{X}$.

By 2.1(1), $S$ has negative curvature if and only if 


$$
Q(X, Y)=\langle R(X, Y) X, Y\rangle \geqslant 0 \quad \text { for all } X, Y \in 8 .
$$

Obviously, the operations $\nabla, R, Q$ are completely determined by $\delta$ and $\langle\cdot, \cdot\rangle$. We shall then say, by abuse of language, that the Lie algebra \&, endowed with the inner product $\langle\cdot, \cdot\rangle$, has negative curvature if (3) holds.

3.2. Whenever we have a Lie algebra endowed with an inner product, we make constant use of the natural identification of $\Lambda^{2} \delta$, the space of alternating 2-tensors on 8 , with $D(8)$ the space of skew-symmetric linear operators on 8. This identification is the unique linear mapping from $\Lambda^{2}$ to $O(\xi)$ which to the elementary tensor $X \wedge Y$ associates the linear endomorphism on defined by

$$
Z \rightarrow\langle X, Z\rangle Y-\langle Y, Z\rangle X \quad \text { for } Z \in \text { 8. }
$$

Moreover, $\Lambda^{2}$ is systematically equipped with the inner product defined, for $X, Y, U, V \in$ \&, by

$$
\langle X \wedge Y, U \wedge V\rangle=\langle X, U X Y, V\rangle-\langle X, V X Y, U\rangle .
$$

In particular if $A \in \mathrm{o}(8) \simeq \Lambda^{2} 8,(4)$ and (5) yield

$$
\langle A, U \wedge=1 / 2\langle A U, V\rangle-1 / 2\langle A V, U\rangle=\langle A U, V\rangle \quad \text { for } U, V \in \delta \text {. }
$$

The covariant differentiation $\nabla$ may then be viewed as a linear map $X \rightarrow \nabla_{X}$ from into $\Lambda^{2} \&$ defined by

$$
\nabla_{X} Y=1 / 2\left((\operatorname{ad} X) Y-(\operatorname{ad} X)^{t} Y-(\operatorname{ad} Y)^{t} X\right) \quad \text { for } X, Y \in \mathbf{8},
$$

where the superscript $t$ denotes transpose with respect to $\langle\cdot, \cdot\rangle$.

The curvature tensor becomes then a symmetric linear endomorphism of $\Lambda^{2} \&$ defined on elementary tensors by

$$
R(X \wedge Y)=\left[\nabla_{X}, \nabla_{Y}\right]-\nabla_{[X, Y]} \quad \text { for } X, Y \in \delta .
$$

The elementary curvature form $Q$ defined by (3) becomes the restriction to elementary tensors of the quadratic form $L \rightarrow\langle R(L), L\rangle$ on $\Lambda^{2}$. Thus for $S, Y \in 8$ we write indifferently

$$
Q(X, Y)=Q(X \wedge Y)=\langle R(X \wedge Y), X \wedge Y\rangle=\langle R(X \wedge Y) X, Y\rangle
$$

using scalar products in $\Lambda^{2} 8$ the first time and in 8 the second time. The fact that $R$ is a symmetric endomorphism of $\Lambda^{2} 8$ may be deduced readily from standard identities (Helgason [5, p. 69]).

We note that a sufficient condition for to have negative curvature is that $R$ be a positive semidefinite linear endomorphism on $\Lambda^{2}$ z. However, easily constructed examples show that this condition is not necessary. In the next two sections, we shall focus on obtaining structural constraints on $z$ and $\langle\cdot, \cdot\rangle$ when 
8 has negative curvature. The following formula for $Q$ will be used repeatedly.

3.3. LemmA. Let 8 be a Lie algebra, $\langle\cdot, \cdot\rangle$ an inner product on 8 , and $R$ the associated curvature tensor. The associated elementary curvature form is, for $S, Y \in \boldsymbol{B}$, given by

$$
\begin{aligned}
\langle R(X \wedge Y), X \wedge Y\rangle= & 3 / 4|(\operatorname{ad} X) Y|^{2}+1 / 2\left\langle(\operatorname{ad} X)^{2} Y, Y\right\rangle+1 / 2\left\langle(\operatorname{ad} Y)^{2} X, X\right\rangle \\
& +\left\langle(\operatorname{ad} X)^{t} X,(\operatorname{ad} Y)^{t} Y\right\rangle-1 / 4\left|(\operatorname{ad} X)^{t} Y+(\operatorname{ad} Y)^{t} X\right|^{2}
\end{aligned}
$$

where $|Z|^{2}=\langle Z, Z\rangle$ for $Z \in \mathbf{8}$.

Proof. This formula occurs elsewhere in the literature in similar forms (e.g., Jensen [8, p. 312] and Heintze [4, p. 4]). The proof consists of straightforward but lengthy calculations using (7) and (8).

3.4. LEMMA. Under the same hypotheses as in Lemma 3.3., we have (11) $\langle R(X \wedge Y), X \wedge Y\rangle=\left\langle(\operatorname{ad} X)^{t} X,(\operatorname{ad} Y)^{t} Y\right\rangle-1 / 4\left|(\operatorname{ad} X)^{t} Y+(\operatorname{ad} Y)^{t} X\right|^{2}$ whenever $X, Y \in \&$ and $[X, Y]=0$, while

$$
\langle R(H \wedge X), H \wedge X\rangle=|(\operatorname{ad} H) X|^{2}-\left|1 / 2\left(\operatorname{ad} H-(\operatorname{ad} H)^{t}\right) X\right|^{2}
$$

whenever $X \in \mathrm{z}$ and $H \in \mathrm{8}$ is orthogonal to $[8,8]$.

Proof. Formula (11) follows trivially from (10), while (12) follows from (10) by easy manipulations and the observation that $H$ orthogonal to [8, 8] implies $(\operatorname{ad} X)^{t} H=0$ for all $X \in \mathbf{8}$. Q.E.D.

3.5. If $(8,\langle\cdot, \cdot\rangle)$ has negative curvature, we know, by Corollary 2.6 , that 8 must be solvable. Hence there exists a nonempty orthogonal complement a to $[\mathrm{z}, \mathrm{z}]=\mathfrak{n}$ in 8 ; for $H \in \mathfrak{a},(12)$ shows that $A=$ ad $H$ must verify $|A X|^{2} \geqslant$ $\left|1 / 2\left(A-A^{t}\right) X\right|^{2}$ for all $X \in \mathfrak{n}$. Trivially, $\{\operatorname{ad} H: H \in \mathfrak{a}\}$ generates a solvable subalgebra of $\mathfrak{g l}(\mathfrak{n})$, the Lie algebra of all linear operators on $\mathfrak{n}$. Our first task is to study, in general, the properties of a vector space $\mathscr{U}$ of linear operators which generates a solvable linear Lie algebra and each of whose elements satisfies the above condition.

4. Almost normal operators.

4.1. Definition. Let $A$ be a linear operator on a finite dimensional real or complex Hilbert space $\mathfrak{\$}$. We say that $A$ is almost normal if

$$
|A v|^{2} \geqslant\left|1 / 2\left(A-A^{*}\right) v\right|^{2} \quad \text { for all } v \in \mathfrak{\$}
$$

where $A^{*}$ is the adjoint of $A$.

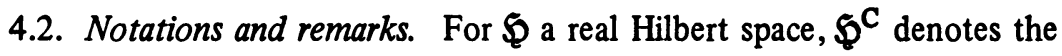
complexification of $\$$ equipped with the complex inner product 


$$
\left\langle v_{1}+i v_{2}, w_{1}+i w_{2}\right\rangle=\left\langle v_{1} w_{1}\right\rangle+\left\langle v_{2} w_{2}\right\rangle+i\left(-\left\langle v_{1}, w_{2}\right\rangle+\left\langle v_{2}, w_{1}\right\rangle\right)
$$

The complexification of a linear operator $A$ on $\mathfrak{Q}$ is the linear operator $A^{\mathrm{C}}$ on $\mathfrak{Q}^{\mathrm{C}}$ defined by $A^{\mathrm{C}}(v+i w)=A v+i A w$. Note that $\left(A^{*}\right)^{\mathrm{C}}=\left(A^{\mathrm{C}}\right)^{*}$. Occasionally, we will abuse notation by continuing to write $A$ in place of $A^{\mathrm{C}}$. An easy computation shows that $A$ is almost normal on $\$$ if and only if $A^{\mathrm{C}}$ is almost normal on $\mathfrak{W}^{\mathbf{C}}$. Although our main interest is in developing information regarding families of almost normal operators on real Hilbert spaces, we shall find it convenient to obtain this information by restriction from the complex setting.

Given a linear operator $A$ on $\mathfrak{W}$, we define

$$
\operatorname{Re} A=1 / 2\left(A+A^{*}\right), \quad \operatorname{Im} A=1 / 2\left(A-A^{*}\right), \quad|A|^{2}=A^{*} A,
$$

$$
N(A)=|A|^{2}-|\operatorname{Im} A|^{2}=(\operatorname{Re} A)^{2}+[\operatorname{Re} A, \operatorname{Im} A]
$$

As usual, for $N$ a selfadjoint operator, the notation $N \geqslant 0$ (respectively, $N>0$ ) means that $N$ is a positive (respectively, positive definite) operator. Obviously, Definition 4.1 may be regarded as saying that $A$ is almost normal if $N(A) \geqslant 0$. Since an operator is normal if and only if $[\operatorname{Re} A, \operatorname{Im} A]=0$, we conclude that every normal operator is almost normal.

The identity operator on a subspace $L$ of $\$$ will be denoted by $I_{L}$. When there is no possibility for confusion (in particular, for $L=\mathfrak{\$}$ ), we shall simply write $I$ in place of $I_{L}$. When $J$ is a skew-symmetric operator commuting with $A$ (for example, $J$ a pure imaginary multiple of $I$ in the complex setting), note that $N(A+J)=N(A)$ and hence $A$ is almost normal if and only if $A+J$ is almost normal. The study of almost normal operators is complicated by the fact that the adjoint of an almost normal operator need not be almost normal. Indeed, let $A$ be the operator on $\mathbf{R}^{3}$ defined by the matrix $\left(a_{i j}\right)_{1<i, j<3}$ with distinct nonzero diagonal elements and $a_{i j}=a_{i i} a_{j j}\left(a_{i i}-a_{j j}\right)^{-1}$ for $i \neq j$. Then $N(A) \geqslant 0$ but $\operatorname{det} N\left(A^{*}\right)<0$.

4.3. Lemma. Let $A$ be an almost normal operator on $\$$ and $L$ an $A$-invariant subspace of $\$$. Then $\left.A\right|_{L}$ is an almost normal operator on $L$. If $\left.A\right|_{L}$ is skewadjoint, then $A$ leaves invariant the orthogonal complement $L^{1}$ of $L$.

Proof. Denote by $P$ the orthogonal projection of $\$$ on $L$; let $A_{1}=P A P$, $A_{2}=(I-P) A(I-P)$, and $Z=P A(I-P)$. With respect to the orthogonal direct sum $\mathfrak{\Phi}=L \oplus L^{\perp}$, we may view $A$ as the matrix of operators

$$
\left[\begin{array}{ll}
A_{1} & Z \\
0 & A_{2}
\end{array}\right] \text {. }
$$

Then 
$N(A)=\left[\begin{array}{ll}A_{1}^{*} & 0 \\ Z^{*} & A_{2}^{*}\end{array}\right]\left[\begin{array}{ll}A_{1} & Z \\ 0 & A_{2}\end{array}\right]+\left[\begin{array}{ll}\operatorname{Im} A_{1} & Z / 2 \\ -Z^{*} / 2 & \operatorname{Im} A_{2}\end{array}\right]^{2}$

$$
=\left[\begin{array}{ll}
N\left(A_{1}\right)-1 / 4\left|Z^{*}\right|^{2} & \left.A_{1}^{*} Z+1 / 2\left(\operatorname{Im} A_{1}\right) Z+Z \operatorname{Im} A_{2}\right) \\
Z^{*} A_{1}-1 / 2\left(Z^{*} \operatorname{Im} A_{1}+\left(\operatorname{Im} A_{2}\right) Z^{*}\right) & N\left(A_{2}\right)+3 / 4|Z|^{2}
\end{array}\right] .
$$

Consequently, $N(A) \geqslant 0$ implies $N\left(A_{1}\right) \geqslant 1 / 4|Z *|^{2} \geqslant 0$ so $A_{1}=\left.A\right|_{L}$ is almost normal. Moreover, if $A_{1}$ is skew-adjoint, $0=N\left(A_{1}\right) \geqslant 1 / 4\left|Z^{*}\right|^{2}$ implies that $Z=$ $Z^{*}=0$ so $A$ leaves $L^{\perp}$ invariant. Q.E.D.

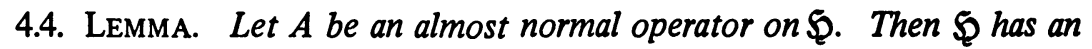
orthogonal decomposition into A-invariant subspaces $\mathfrak{\bigvee}_{0}$ and $\mathfrak{Q}_{1}$ where $\left.A\right|_{\mathfrak{\Phi}_{0}}$ is skew-adjoint and the eigenvalues of $\left.A\right|_{\Phi_{1}}$ have nonzero real parts.

Proof. From the remarks made in 4.2 , we may assume that $\$$ is a complex Hilbert space. Suppose that $v$ is a nonzero vector in $\mathfrak{S}$ and $\lambda$ a pure imaginary scalar such that $(A-\lambda)^{k} v=0$ for some integer $k>1$. Set $v_{1}=(A-\lambda)^{k-1} v$ and $v_{2}=(A-\lambda I)^{k-2} v$. Suppose $v_{1} \neq 0$. Since $A v_{1}=\lambda v_{1}$, the restriction of $A$ to the one-dimensional space spanned by $v_{1}$ is skew-adjoint. By Lemma 4.3, $A$ leaves invariant the space of vectors orthogonal to $v_{1}$. Hence

$$
\begin{aligned}
0 & =\left\langle(A-\lambda D)\left(v_{2}-\left\langle v_{2}, v_{1}\right\rangle v_{1} /\left\|v_{1}\right\|^{2}\right), v_{1}\right\rangle \\
& =\left\langle(A-\lambda I) v_{2}, v_{1}\right\rangle=\left\langle v_{1}, v_{1}\right\rangle
\end{aligned}
$$

which is a contradiction. Therefore $v_{1}=0$ and by a trivial induction, $A v=\lambda v$.

Now define $\mathfrak{Q}_{0}$ as the sum of generalized eigenspaces corresponding to pure imaginary eigenvalues of $A$. Lemma 4.3 and the argument just given imply that $\mathfrak{W}_{0}$ is an orthogonal direct sum of eigenspaces. Therefore $\left.A\right|_{\$_{0}}$ is skew-adjoint so another application of Lemma 4.3 implies that $\bigvee_{1}=\$_{0}^{1}$ is $A$-invariant. Clearly, $\tilde{\bigotimes}_{1}$ is the sum of generalized eigenspaces corresponding to eigenvalues of $A$ with nonzero parts. Q.E.D.

4.5. We remark that Lemma 4.4 provides the following characterization of almost normal operators. Let $A$ be a linear operator on a finite dimensional real or complex vector space $V$. By the primary decomposition theorem, $V$ has the direct sum decomposition $V=V_{0}+V_{1}$ where $V_{0}$ is the sum of generalized eigenspaces corresponding to pure imaginary eigenvalues of $A$ and $V_{1}$ is the sum of generalized eigenspaces corresponding to eigenvalues of $A$ with nonzero real part. Then $V$ admits an inner product relative to which $A$ is almost normal if and only if $\left.A\right|_{V_{0}}$ is semisimple. We just proved the direct statement. For the converse, one may construct an inner product on $V$ as follows. Take any inner product on $V_{0}$ for which $\left.A\right|_{V_{0}}$ is skew-symmetric. After complexification if necessary, take any basis $v_{1}, \ldots, v_{n}$ of $V_{1}$ in which the matrix of $\left.A\right|_{V_{1}}$ is upper 
triangular. In the basis $d_{1} v_{1}, \ldots, d_{n} v_{n}$ where $d_{1}>\cdots>d_{n}$, the matrix of $\left.A\right|_{V_{1}}$ will have arbitrarily small off diagonal entries for proper choices of the $d_{i}$. Hence, for suitable choices of the $d_{i},\left.A\right|_{V_{1}}$ will be almost normal relative to the inner product on $V_{1}$ for which $d_{1} v_{1}, \ldots, d_{n} v_{n}$ is an orthonormal basis. The definition of the inner product on $V$ is completed by taking $V_{0}$ and $V_{1}$ orthogonal.

4.6. LEMma. Let $A$ be an almost normal operator on $\$$, L an A-invariant subspace of $\$, P$ the orthogonal projection of $A$ on $L$. Suppose that the operator $(I-P) A(I-P)$ is skew-adjoint. Then $A$ leaves $L^{1}$ invariant.

Proof. First consider the following special case: $A_{1}=\left.A\right|_{L}$ is invertible on $L$ and $A_{2}=(I-P) A(I-P)=0$. Then for any nonzero vector $v \in L^{1}, A v \in L$, so there exists a vector $w \in L$ such that $A v=A w$. By Lemma 4.3, the space orthogonal to $(w-v)$ is left-invariant by $A$. Consequently, for $u=A_{1}^{-1} w \in L$,

$$
\begin{aligned}
\|w\|^{2} & =\left\langle w-v, A_{1} u\right\rangle \\
& =\left\langle w-v, A\left(u-\left(\langle u, w-v\rangle /\|w-v\|^{2}\right)(w-v)\right)\right\rangle=0 .
\end{aligned}
$$

Thus $A v=0$ and we conclude that $\left.A\right|_{\perp \perp}=0$.

For the general case, we may assume as before that $\$$ is complex. Then the hypothesis that $A_{2}$ is skew-adjoint implies that $L^{\perp}$ is spanned by nonzero vectors $v$ with the property that there exists a pure imaginary scalar $\lambda$ such that $A v-\lambda v \in L$. Fix such a vector $v$ and let $\mathfrak{W}^{\prime}$ denote the $A$-cyclic subspace of $\mathfrak{\$}$ generated by $v$, i.e. $\mathfrak{S}^{\prime}=\operatorname{span}\left\{A^{k} v: k \geqslant 0\right\}$. By Lemma $4.3,\left.A\right|_{\mathfrak{S}^{\prime}}$ is an almost normal operator on $\mathfrak{\Phi}^{\prime}$. By the remarks made in 4.2 , the operator $B=\left.A\right|_{\mathfrak{Q}^{\prime}}-$ $\lambda I_{\mathfrak{Q}^{\prime}}$ is almost normal on $\mathfrak{Q}^{\prime}$. By definition, $B$ leaves $L^{\prime}=\mathfrak{W}^{\prime} \cap L$ invariant and $B v \in L^{\prime}$. We now claim that $\left.B\right|_{L^{\prime}}$ is invertible. An easy induction on $n$ shows that if $B v \neq 0$, the intersection of $L$ with the span of $\left\{A^{k} v: 0 \leqslant k \leqslant n\right\}$ is the span of $\left\{B^{k} v: 1 \leqslant k \leqslant n\right\}$. Hence there is an $n$ such that $L^{\prime}$ is the span of $\left\{B^{k} v: 1 \leqslant k \leqslant n\right\}$. Assume that for some $w=\Sigma_{1 \leqslant k \leqslant n} c_{k} B^{k} v$, we have $B w=0$. Then

$$
0=B w=B^{2}\left(\sum_{1<k<n} c_{k} B^{k-1} v\right) .
$$

Using Lemma 4.4, we get $B\left(\Sigma_{1<k<n} c_{k} B^{k-1} v\right)=0$, i.e. $w=0$. Hence the hypotheses for the special case are verified, and we conclude that $B v=0$, i.e. $A v=$ $\lambda v$. It follows that $A$ leaves $L^{1}$ invariant. Q.E.D.

4.7. Definition. Let $\mathfrak{A}$ be a finite-dimensional real vector space, $\mathfrak{A}^{*}$ its dual, $\left(\mathscr{Q}^{*}\right)^{\mathbf{C}}$ the space of complex valued linear functionals on $\mathscr{\mathscr { H }}$. When $\mu \in \mathfrak{Q}^{*}$, ker $\mu$ denotes the kernel of $\mu$. We say that two elements $\lambda_{1}=\mu_{1}+i \nu_{1}, \lambda_{2}=$ $\mu_{2}+i \nu_{2}$ in $\left(\mathscr{Q}^{*}\right)^{\mathrm{C}}$ are equivalent (denoted $\lambda_{1} \sim \lambda_{2}$ ) if ker $\mu_{1}=\operatorname{ker} \mu_{2} \subseteq$ $\operatorname{ker}\left(\nu_{2}-\nu_{1}\right)$. 
Obviously, this defines an equivalence relation on $\left(\mathscr{Q}^{*}\right)^{\mathbf{C}}$. Note that $\mu_{1}+$ $i \nu_{1} \sim \mu_{2}+i \nu_{2}$ if and only if $\mu_{1}=c \mu_{2}$ and $\nu_{1}-\nu_{2}=d \mu_{2}$ where $c, d$ are real scalars with $c \neq 0$.

4.8. THEOREM. Let $\mathfrak{A}$ be a real vector space of linear operators of a

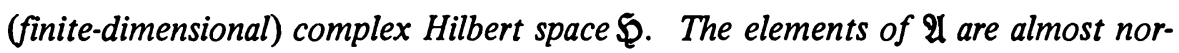
mal and the Lie algebra generated by $\mathfrak{A}$ is solvable if and only if there exist $\mathfrak{U}-$ invariant subspaces $\mathfrak{\Phi}_{p}, 1 \leqslant p \leqslant m$, of $\mathfrak{W}$, and elements $\lambda_{p}=\mu_{p}+i v_{p}, 1 \leqslant p \leqslant m$, in $\left(\mathscr{U}^{*}\right)^{\mathrm{C}}$ such that

(i) $\mathfrak{Q}=\bigoplus_{1 \leqslant p \leqslant m} \mathfrak{Q}_{p}$,

(ii) for each $p=1, \ldots, m$ there is on $\mathfrak{W}_{p}$ an almost normal operator $E_{p}$ whose eigenvalues have nonzero real parts and such that, for all $A \in \mathfrak{U},\left.A\right|_{\mathfrak{P}_{p}}=$ $\mu_{p}(A) E_{p}+i \nu_{p}(A) I_{\$_{p}}$.

It is possible to choose the decomposition in such a way that the equivalence classes (Definition 4.7) of the $\lambda_{p}$ in $\left(\mathscr{A}^{*}\right)^{\mathrm{C}}$ are distinct; under this extra condition, the subspaces $\aleph_{p}$ and the equivalence classes of the $\lambda_{p}$ are uniquely determined by $\mathcal{A}$ (up to reordering).

Proof. The sufficiency is obvious since if (i) and (ii) hold, $\mathscr{U}$ is clearly an abelian subalgebra of $\mathfrak{g l}(\mathfrak{\$})$, and $N\left(\left.A\right|_{\mathfrak{\Phi}_{p}}\right)=\mu_{p}(A)^{2} N\left(E_{p}\right) \geqslant 0$ (see Remarks 4.2). The necessity will be proved by induction on $n=\operatorname{dim} \$$, the case $n=1$ being trivial. By Lie's theorem for solvable linear Lie algebras [5, p. 134] there exists an $\mathscr{U}$-invariant subspace $L$ in $\$$ having codimension 1. By Lemma 4.3, $\left\{\left.A\right|_{L}\right.$ : $A \in \mathscr{U}\}$ is a vector space of almost normal operators on $L$. By the induction hypothesis, we find for $p=1, \ldots, m$, subspaces $L_{p}$ of $L$, pairwise nonequivalent elements $\lambda_{p}=\mu_{p}+i \nu_{p}$ in $\left(\mathscr{Q}^{*}\right)^{\mathrm{C}}$ and operators $E_{p}$ on $L_{p}$ verifying (i) and (ii).

Define $Z_{p}(A)=P_{p} A Q$ where $P_{p}$ is the orthogonal projection of $\$$ on $L_{p}$ and $Q=I-\Sigma_{p} P_{p}$ is the projection on the one-dimensional subspace $L^{\perp}$. Whenever $\mu_{p}(A)=0,\left.A\right|_{L_{p}}$ is skew-symmetric by (ii), and Lemma 4.3 implies that $A\left(L^{\perp}\right)$ is orthogonal to $L_{p}$; hence $\mu_{p}(A)=0$ implies $Z_{p}(A)=0$. Since $A \rightarrow$ $Z_{p}(A)$ is linear and vanishes on ker $\mu_{p}$, we may write $Z_{p}(A)=\mu_{p}(A) Z_{p}$ for some fixed map $Z_{p}$ from $L^{\perp}$ into $L_{p}$. Clearly, $Z_{p}$ is uniquely determined if $\mu_{p} \not \equiv 0$. For $\mu_{p} \equiv 0$, we take $Z_{p}=0$. On the other hand, by linearity of $A \rightarrow Q A Q$, there is a unique $\lambda=\mu+i \nu$ in $\left(\mathscr{Q}^{*}\right)^{\mathrm{C}}$ such that $Q A Q=(\mu(A)+i \nu(A)) I_{L^{\perp}}$.

Assume first that $Z_{p}=0$ for all $p$. If $\lambda \sim \lambda_{p}$ for some $p$, define $\mathfrak{\Phi}_{p}=L_{q}$ for $q \neq p, \mathfrak{Q}_{p}=L_{p} \oplus L^{\perp}$, and replace $E_{p}$ by $E_{p}+(c+i d) Q$ where $\mu=c \mu_{p}$, $\nu=\nu_{p}+d \mu_{p}$. If $\lambda$ is not equivalent to any of the $\lambda_{p}$, define $\bigvee_{p}=L_{p}$ for $1 \leqslant p \leqslant m, \mathfrak{\Phi}_{m+1}=L^{\perp}, \lambda_{m+1}=\lambda$, and $E_{m+1}=I_{L \perp}$. In both cases trivial verifications show that (i) and (ii) still hold.

Assume now that for some $p, Z_{p} \neq 0$. By Lemma 4.6, $\mu_{p}(A) Z_{p}=0$ 
whenever $\mu(A)=0$. Consequently, both $\mu_{p}$ and $\mu$ are nonzero, and $\mu=c \mu_{p}$ for some scalar $c$. We will show below that $\operatorname{ker} \mu_{p} \subseteq \operatorname{ker}\left(\nu-\nu_{p}\right)$ and hence $\lambda \sim \lambda_{p}$. Assuming this has been shown, we must have $Z_{q}=0$ for all $q \neq p$ since the $\lambda_{p}$ are in distinct equivalence classes. The induction step is completed as above by replacing $L_{p}$ by $\mathfrak{\bigotimes}_{p}=L_{p} \oplus L^{1}$ with $E_{p}$ now replaced by $E_{p}+Z_{p}+(c+i d) Q$ where $\nu=\nu_{p}+d \mu_{p}$.

We now prove the claim $\lambda \sim \lambda_{p}$. Fix $A_{1}$ in $\mathfrak{A}$ with $\mu_{p}(A)=1$ and let $A_{0} \in$ ker $\mu_{p}$. For $t \in \mathrm{R}$ define $N_{p}(t)=\left(P_{p}+Q\right) N\left(A_{1}+t A_{0}\right)\left(P_{p}+Q\right)$. Regard $N_{p}(t)$ as an operator on $L_{p} \oplus L^{\perp}$. Since $\mu\left(A_{0}\right)=0$ implies $Z_{q}\left(A_{0}\right)=0$ for all $q$, formula (2) implies that $N_{p}(t)$ is represented by the matrix of operators

$$
N_{p}(t)=\left[\begin{array}{ll}
N\left(E_{p}\right)-\frac{1}{4}\left|Z_{p}^{*}\right|^{2} & B_{p} Z_{p}+i \frac{t}{2}\left(\nu-\nu_{p}\right)\left(A_{0}\right) Z_{p} \\
Z_{p}^{*} B_{p}^{*}-i \frac{t}{2}\left(\nu-\nu_{p}\right)\left(A_{0}\right) Z_{p}^{*} & c^{2} Q+\frac{3}{4} \sum_{q=1}^{m}\left|Z_{q}\left(A_{1}\right)\right|^{2}
\end{array}\right]
$$

where $B_{p}=\operatorname{Re} E_{p}-1 / 2 \operatorname{Im} E_{p}+i\left(\nu-v_{p}\right)\left(A_{1}\right) I_{L_{p}} / 2$. By hypothesis, $N_{p}(t) \geqslant 0$ for all $t$. But $N_{p}(t)=N_{p}(0)+t W_{p}$ where $W_{p} \stackrel{p}{=} i\left(\nu-\nu_{p}\right)\left(A_{0}\right)\left(Z_{p}-Z_{p}^{*}\right) / 2$. Clearly this is possible only if $\left\langle W_{p} v, v\right\rangle=0$ for all $v \in L_{p} \oplus L^{\perp}$. Since $W_{p}$ is selfadjoint, we conclude that $W_{p}=0$; on the other hand, $Z_{p}$ is a nonzero map from $L^{\perp}$ to $L_{p}$ so that $Z_{p}-Z_{p}^{*}$ is nonzero, and $W_{p}=0$ implies $\nu\left(A_{0}\right)=\nu_{p}\left(A_{0}\right)$. We have thus demonstrated our claim $\operatorname{ker} \mu_{p} \subset \operatorname{ker}\left(\nu-\nu_{p}\right)$ and the existence of the decomposition described in Theorem 4.8 is proved. Its uniqueness will be an obvious consequence of the following corollary. Q.E.D.

For $\mathfrak{A}, \mathfrak{Q}$ as in Theorem 4.8 and $\lambda \in\left(\mathfrak{Q}^{*}\right)^{\mathrm{C}}$, define $\mathfrak{W}_{\lambda}=\{v \in \mathfrak{Q}:$ for some $\left.k \geqslant 1(A-\lambda(A))^{k} v=0\right\}$. If $\mathfrak{Q}_{\lambda} \neq 0, \lambda$ is called a root of $\mathscr{Q}$ and $\mathfrak{W}_{\lambda}$ a root space of $\mathfrak{A}$.

4.9. COROLlaRY. Let $\mathfrak{A}$ be a real vector space of almost normal endomorphisms of a complex Hilbert space $\$$. Assume the linear Lie algebra generated by $\mathfrak{A}$ in $\mathfrak{g l}(\mathfrak{Q})$ is solvable. Then $\mathfrak{A}$ is abelian and hence $\mathfrak{W}$ is a direct sum of root spaces of $\mathfrak{A}$. Root spaces $\mathfrak{W}_{\lambda}$ and $\mathfrak{W}_{\lambda^{\prime}}$ are orthogonal whenever $\lambda$ is not equivalent to $\lambda^{\prime}$. The unique decomposition of $\$$ described in Theorem 4.8 is given by $\mathfrak{W}_{p}=\Sigma_{\lambda \sim \lambda_{p}} \mathfrak{W}_{\lambda}$ where the elements $\lambda_{p}, 1 \leqslant p \leqslant m$, are pairwise nonequivalent in $\left(\mathfrak{Q}^{*}\right)^{\mathrm{C}}$. Finally, if $\lambda \in\left(\mathfrak{Q}^{*}\right)^{\mathrm{C}}$ is purely imaginary, we have $\left.A\right|_{\mathfrak{P}_{\lambda}}=\lambda(A) I$ for all $A \in \mathfrak{A}$.

PROof. In view of Theorem 4.8(ii), $\mathfrak{U}$ is obviously abelian. Starting from a decomposition $\mathfrak{Q}=\bigoplus_{p} \mathfrak{W}_{p}$ corresponding to pairwise nonequivalent $\lambda_{p} \in\left(\mathscr{U}^{*}\right)^{\mathbf{C}}$, we get a root space decomposition of $\mathfrak{\$}$ relative to $\mathfrak{A}$ by finding a primary decomposition for each subspace $\mathfrak{\bigotimes}_{p}$ relative to the action of $E_{p}$. Thus for each 
eigenvalue $c+i d$ of $E_{p}$, we get the root $\lambda=c \mu_{p}+i\left(d \mu_{p}+\nu_{p}\right) \sim \lambda_{p}$ of $\mathfrak{A}$. It is then obvious that $\mathfrak{W}_{p}=\Sigma_{\lambda \sim \lambda_{p}} \mathfrak{W}_{\lambda}$ which proves simultaneously the uniqueness (up to permutation) of the equivalence classes of the $\lambda_{p}$, and of the subspaces $\mathfrak{W}_{p}$. Moreover, if $\lambda$ is not equivalent to $\lambda^{\prime}, \mathfrak{W}_{\lambda}$ and $\mathfrak{W}_{\lambda^{\prime}}$ lie in distinct subspaces $\mathfrak{W}_{p}$ and $\mathfrak{W}_{q}$ and hence must be orthogonal. The last statement in Corollary 4.9 is a consequence of Lemma 4.4. Q.E.D.

It is easy to translate the results of Theorem 4.8 and Corollary 4.9 to the case when $\$$ is a real Hilbert space. We shall do it in the next section in the particular context of solvable Lie algebras.

5. Structure of solvable algebras with negative curvature.

5.1. Notations. Let be a (real) solvable Lie algebra. Denote by $\mathfrak{n}=$ $[8,8]$ the derived Lie algebra of 8 . As Theorem 5.2 will show, in the situation of interest to us, 8 will have, as a vector space, a direct sum decomposition $=$ $\mathfrak{a}+\mathfrak{n}$ with an abelian action of $\mathfrak{a}$ on $\mathfrak{n}$. Whenever we have such a decomposition, we define, as usual, for $\lambda \in\left(\mathfrak{a}^{*}\right)^{\mathbf{C}}$ the subspaces $\mathfrak{n}_{\lambda}^{\mathbf{C}}$ of $\mathfrak{n}^{\mathrm{C}}$ by

$$
\mathfrak{n}_{\lambda}^{\mathrm{C}}=\left\{X \in \mathfrak{n} \mid(\operatorname{ad} H-\lambda(H))^{k} X=0 \text { for some } k \geqslant 1 \text { and all } H \in \mathfrak{a}\right\} \text {. }
$$

We call $\lambda$ a root of $a$ in $\mathfrak{n}$ whenever $\mathfrak{n}_{\lambda}^{\mathbb{C}} \neq 0$ and call $\mathfrak{n}_{\lambda}^{\mathbb{C}}$ the associated root space of $\boldsymbol{a}$ in $\mathfrak{n}$. Then $\mathfrak{n}^{\mathbf{c}}$ is the direct sum of the root spaces $\mathfrak{n}_{\lambda}^{\mathbf{C}}$.

For $\lambda=\alpha+i \beta \in\left(\mathfrak{a}^{*}\right)^{\mathbf{C}}$, we define $\mathfrak{n}_{\alpha, \beta}=\mathfrak{n} \cap\left(\mathfrak{n}_{\lambda}^{\mathrm{C}}+\mathfrak{n} \frac{\mathrm{C}}{\lambda}\right)=\mathfrak{n}_{\alpha,-\beta}$. If $(\alpha \pm i \beta)$ is a root of $a$ in $\mathfrak{n}$, we call $\mathfrak{n}_{\alpha, \beta}$ a generalized root space of $a$ in $\mathfrak{n}$. Clearly, $\mathfrak{n}$ is the direct sum of the generalized root spaces $\mathfrak{n}_{\alpha, \beta}$.

$\$ 4$ has shown the interest of the following equivalence relation on $\left(a^{*}\right)^{c}$ : for $\lambda=\alpha+i \beta, \mu=\gamma+i \delta$, in $\left(a^{*}\right)^{\mathrm{C}}$ we say that $\lambda$ is equivalent to $\mu(\lambda \sim \mu)$ whenever $\operatorname{ker} \alpha=\operatorname{ker} \gamma \subseteq \operatorname{ker}(\beta-\delta)$.

5.2. THEOREM. Let be a Lie algebra endowed with an inner product. Assume that has negative curvature. Then is solvable and the orthogonal complement $a$ of $\mathfrak{n}=[8,8]$ in 8 is abelian; moreover, for all $H \in \mathfrak{a}$, ad $H$ and $(\operatorname{ad} H)^{\mathbf{C}}$ are almost normal on $\mathfrak{n}$ and $\mathfrak{n}^{\mathrm{C}}$, respectively. In particular, the conclusions of Theorem 4.8 and Corollary 4.9 apply to the operators $(\mathrm{ad} H)^{\mathrm{C}}$, $H \in a .{ }^{2}$ )

Proof. We have seen in 3.5 that 8 must be solvable and that the operators ad $H, H \in a$, are almost normal on $\mathfrak{n}$. Then, by Remarks 4.2, (ad $H)^{\mathrm{C}}$ is almost normal on $\mathfrak{n}^{\mathbf{C}}$. The subalgebra of $\mathfrak{g l}^{l}\left(\mathfrak{n}^{\mathbf{C}}\right)$ generated by $\mathfrak{A}=\left\{(\operatorname{ad} H)^{\mathrm{C}}, H \in \mathfrak{a}\right\}$ is obviously solvable since 8 is solvable. Hence Theorem 4.8 and Corollary 4.9 apply to $\mathfrak{A}$ and $\mathfrak{n}^{\mathbf{C}}$. In particular $\mathfrak{A}$ is abelian. Hence $\mathfrak{n}$ has a generalized root

(2) The authors wish to thank the reviewer for the following information: "Commutativity of a was independently proved by Ernst Heintze at the end of 1973. Heintze did not publish, but he showed the result to some people at Berkeley". 
space decomposition $\mathfrak{n}=\Sigma \mathfrak{n}_{\alpha, \beta}$. Fix a root $\alpha+i \beta=\lambda$ of $\mathfrak{a}$ in $\mathfrak{n}$, and suppose $H \in \operatorname{ker} \alpha$. Thus ad $\left.H\right|_{\mathfrak{n}_{\alpha, \beta}}$ has purely imaginary eigenvalues and is almost normal (Lemma 4.3); it must then be skew-symmetric by Lemma 4.4.

Calling $Q$ the orthogonal projection of 8 on the orthogonal complement of $\mathfrak{n}_{\alpha, \beta}$, formula $3.4(12)$ for the elementary curvature form yields then, for $X \in \mathfrak{n}_{\alpha, \beta}$,

$$
\langle R(H \wedge X), H \wedge X\rangle=-1 / 4\left|Q \circ(\operatorname{ad} H)^{t} X\right|^{2}
$$

and hence, by negativity of the sectional curvature, $Q \circ(\operatorname{ad} H)^{t} X=0$. In particular this implies that $[H, K]$ is orthogonal to $\mathfrak{n}_{\alpha, \beta}$ for all $K \in \mathfrak{a}$. Since the bracket operation is alternating bilinear and ker $\alpha$ has codimension $\leqslant 1$, we conclude that $[\mathfrak{a}, \mathfrak{a}]$ is orthogonal to $\mathfrak{n}_{\alpha, \beta}$. Since $\mathfrak{n}=\Sigma \mathfrak{n}_{\alpha, \beta},[\mathfrak{a}, \mathfrak{a}]$ is orthogonal to the derived subalgebra $\mathfrak{n}$ of $\boldsymbol{z}$ and hence is $\{0\}$, i.e. $a$ is abelian. Q.E.D.

5.3. Consequences. Let $z=a \oplus \mathfrak{n}$ be a solvable Lie algebra with negative curvature as in Theorem 5.2.

Let $\alpha_{0}=0$. We select and fix elements $\alpha_{1}, \alpha_{2}, \ldots, \alpha_{m}$ in $a^{*}$ such that the real part of any root of $a$ in $\mathfrak{n}$ is a nonzero multiple of some unique $\alpha_{j}$ $(0 \leqslant j \leqslant m)$. Next, we choose a finite set of pairwise nonequivalent elements $\mu_{p}+i v_{p}$ in $\left(a^{*}\right)^{C}$ satisfying the following conditions:

each root of $a$ is equivalent to $\mu_{p}+i \nu_{p}$ for some unique index $p$;

each $\mu_{p}$ is equal to $\alpha_{j}$ for some $j(0 \leqslant j \leqslant m)$;

$\mu_{p}$ and $\nu_{p}$ are independent whenever both are nonzero;

if $\nu_{p} \neq 0$, there exists an index $q$ such that $\mu_{q}=\mu_{p}, \nu_{q}=-\nu_{p}$. The existence of such a set follows easily from the definition of equivalence given in 4.7 .

(i) Denote by $\mathfrak{\Phi}_{p} \subset \mathfrak{n}^{\mathbf{C}}$ the sum of all root spaces $n_{\lambda}^{\mathrm{C}}$ with $\lambda \sim \mu_{p}+i \nu_{p}$. By Theorem 4.8 and Corollary 4.9 we have the orthogonal direct sum decomposition $\mathfrak{n}^{\mathbf{C}}=\bigoplus_{p} \mathfrak{\bigvee}_{p} ;$ moreover, there exists an almost normal linear operator $E$ on $\mathfrak{n}^{\mathrm{C}}$ such that for all $p$ and all $H \in \mathfrak{a}, E$ leaves $\mathfrak{\Phi}_{p}$ invariant and ad $\left.H\right|_{\mathfrak{\Phi}_{p}}=$ $\left.\mu_{p}(H) E\right|_{\mathfrak{\Phi}_{p}}+i \nu_{p}(H) I$. If $\mu_{p}=0$, the restriction of $E$ to $\$_{p}$ is clearly arbitrary; we take it to be zero. Otherwise, $\left.E\right|_{b_{p}}$ is uniquely determined and all of its eigenvalues have nonzero real parts. Obviously, $E$ commutes with the action of $a$ and leaves $\mathfrak{n}$ invariant. In particular, the subspaces $\mathfrak{n}_{\alpha, \beta}$ and $\mathfrak{n}_{\lambda}^{\mathbf{C}}$ are $E$-invariant.

(ii) From (i), it follows that for any root $\lambda$ of $a$ in $\mathfrak{n}$, the image of $\mathfrak{n}_{\lambda}^{\mathbf{C}}$ under $E^{*}$ or $\operatorname{Re}(E)$ is contained in the sum of root spaces $\mathfrak{n}_{\nu}^{\mathrm{C}}$ for which $\nu$ is equivalent to $\lambda$.

(iii) Two root spaces $n_{\lambda}^{\mathbf{C}}$ and $\mathfrak{n}_{\mu}^{\mathbf{C}}$ are orthogonal whenever $\lambda$ is not equivalent to $\mu$. In particular $n_{\alpha, \beta}$ and $n_{\gamma, \delta}$ are orthogonal whenever $\gamma$ and $\alpha$ are independent.

(iv) Let $\lambda=\alpha+i \beta$ be any root of $\mathfrak{a}$ in $\mathfrak{n}$. Write $\alpha=c \alpha_{j}$ for some $c \neq 0$, $j=0,1, \ldots, m$. Then, there exists $d \in \mathbf{R}$ such that, for all $H \in a$, 


$$
\text { ad }\left.H\right|_{n_{\lambda}}=\left.\alpha_{j}(H) E\right|_{n}{ }_{\lambda}^{c}+i\left(\beta(H)+d \alpha_{j}(H)\right) I .
$$

(v) For $j=0,1, \ldots, m$, let $\mathfrak{n}_{j} \subset \mathfrak{n}$ be the sum of all generalized root spaces $\mathfrak{n}_{\alpha, \beta}$ for which $\alpha$ is a nonzero multiple of $\alpha_{j}$. Thus $\mathfrak{n}_{j}^{\mathrm{C}}=\Sigma_{\mu_{p}=\alpha_{j}} \mathfrak{\bigotimes}_{p}$ and $\mathfrak{n}=\bigoplus_{0 \leqslant j \leqslant m} \mathfrak{n}_{j}$ as an orthogonal sum of vector spaces. For all $j$ and all $H \in \mathfrak{n}$, $\mathfrak{n}_{j}$ is invariant under $E, E^{t}$, ad $H$, and (ad $\left.H\right)^{t}$. Let $E_{j}$ be the composition of $E$ with orthogonal projection from $\mathfrak{n}$ onto $\mathfrak{n}_{j}$. For $H \in \mathfrak{n}$, the expression for ad $H$ given in 5.3(i) implies that the operator ad $H-\Sigma_{1<j<m} \alpha_{j}(H) E_{j}$ is skew-symmetric.

(vi) The following notations will be used frequently. We set $n_{+}=$ $\bigoplus_{1<j \leqslant m} \mathfrak{n}_{j}$; hence $\mathfrak{n}=\mathfrak{n}_{0} \oplus \mathfrak{n}_{+}$.

We denote by $\Delta$ the (finite) set of all $\alpha \in n^{*}$ such that, for some $\beta \in a^{*}$, $\alpha+i \beta$ is a root of $\boldsymbol{a}$ in $\mathfrak{n}$.

For $\alpha \in a^{*}$, we call $H_{\alpha}$ the unique vector in a such that $\left\langle H_{\alpha}, H\right\rangle=\alpha(H)$ for all $H \in \mathfrak{a}$. We set $\langle\alpha, \beta\rangle=\left\langle H_{\alpha}, H_{\beta}\right\rangle$ and $|\alpha|^{2}=\langle\alpha, \alpha\rangle$ for $\alpha, \beta \in a^{*}$.

5.4. LEMMA. Let 8 be a solvable Lie algebra endowed with a scalar product $\langle\cdot, \cdot\rangle$. If has negative curvature, we have (using the notations of 5.1 and 5.3):

(i) $(\operatorname{ad} X)^{t} X \in \mathfrak{n}$ for all $X \in \mathfrak{n}_{0}$;

(ii) for all $\alpha \in \Delta, X \in \mathfrak{n}_{\alpha, \beta}, H \in \mathfrak{a}$,

$$
\left\langle(\operatorname{ad} X)^{t} X, H\right\rangle=-\alpha(H)|\alpha|^{-2}\left\langle\left[H_{\alpha}, X\right], X\right\rangle ;
$$

(iii) in any a-invariant subspace of $n_{\alpha, \beta}$ there exists a vector $X$ such that $\left\langle\left[H_{\alpha}, X\right], X\right\rangle>0$;

(iv) if $\alpha \in \Delta$ and $\mathfrak{n}_{\alpha, \beta} \cap \mathcal{z} \neq 0$ (where $z$ is the center of $\mathfrak{n}$ ), then $\langle\alpha, \gamma\rangle$ $\geqslant 0$ for all $\gamma \in \Delta$.

Proof. Let $X \in \mathfrak{n}_{\alpha, \beta}$ and $H \in \operatorname{ker} \alpha$. Then, by Lemma 4.4 , ad $\left.H\right|_{\mathfrak{n}_{\alpha, \beta}}$ is skew-symmetric, so $\left\langle(\operatorname{ad} X)^{t} X, H\right\rangle=-\langle X,(\operatorname{ad} H) X\rangle=0$. In particular, taking $\alpha=0$ and $\beta$ arbitrary in $a^{*}$, we get (i). For $\alpha \in \Delta, X \in \mathfrak{n}_{\alpha, \beta}$ we see that $(\operatorname{ad} X)^{t} X$ is orthogonal to $\operatorname{ker} \alpha$, i.e. $P_{a}\left((\operatorname{ad} X)^{t} X\right)$ is a multiple of $H_{\alpha}$, where $P_{a}$ is the orthogonal projection of $z$ on $\boldsymbol{a}$. Hence

$$
P_{a}\left((\operatorname{ad} X)^{t} X\right)=|\alpha|^{-2}\left\langle(\operatorname{ad} X)^{t} X, H_{\alpha}\right\rangle H_{\alpha}=-|\alpha|^{-2}\left\langle\left[H_{\alpha}, X\right], X\right\rangle H_{\alpha}
$$

from which (ii) follows.

Now let 0 be an $a$-invariant subspace of $\mathfrak{n}_{\alpha, \beta}$. Since $a$ is abelian, we may find $Z=X+i Y$ in ${ }^{C}{ }^{C} \cap \mathfrak{n}_{\alpha+i \beta}^{\mathbf{C}}$ such that $(\operatorname{ad} H)^{\mathbf{C}} Z=(\alpha(H)+i \beta(H)) Z$ for all $H \in a$. Hence

$$
\left\langle\left[H_{\alpha}, X\right], X\right\rangle=|\alpha|^{2}|X|^{2}-\langle\alpha, \beta X X, Y\rangle, \quad\left\langle\left[H_{\alpha}, Y\right], Y\right\rangle=|\alpha|^{2}|Y|^{2}+\langle\alpha, \beta X X, Y\rangle .
$$

At least one of these expressions is strictly positive and (iii) follows.

Now $\mathfrak{n}_{\alpha, \beta} \cap \mathfrak{z}$ is $a$-invariant since $a$ acts by derivations on $\mathfrak{n}$. Given $\gamma \in \Delta$ with $\mathfrak{n}_{\gamma, \delta} \neq 0$ we use (iii) to find vectors $X \in \mathfrak{n}_{\alpha, \beta} \cap z$ and $Y \in \mathfrak{n}_{\gamma, \delta}$ such that

$$
P_{a}\left((\operatorname{ad} X)^{t} X\right)=-c H_{\alpha}, \quad P_{a}\left((\operatorname{ad} Y)^{t} Y\right)=-d H_{\gamma},
$$


with $c$ and $d$ strictly positive. Since $X \in \mathcal{z},(\operatorname{ad} X)^{t} X$ is in a. The curvature being negative, formula $3.4(11)$ yields

$$
c d\langle\alpha, \gamma\rangle=\left\langle(\operatorname{ad} X)^{t} X,(\operatorname{ad} Y)^{t} Y\right) \geqslant\langle R(X \wedge Y), X \wedge Y\rangle \geqslant 0
$$

and hence $\langle\alpha, \gamma\rangle \geqslant 0$. Q.E.D.

5.5. Proposition. Let 8 be a solvable Lie algebra endowed with a scalar product. Assume 8 has negative curvature. Then (using the notations of 5.1, 5.3) $\mathfrak{n}_{0}$ is contained in the center of $\mathfrak{n}$ and $[\mathfrak{n}, \mathfrak{n}]$ is contained in $\mathfrak{n}_{+}=$ $\Sigma_{1 \leqslant j \leqslant m} \mathfrak{n}_{j}$ In particular, $\mathfrak{n}_{0}$ is an abelian subalgebra of 8 and $\mathfrak{n}_{+}$is an ideal of 8 .

Proof. Let $\mathfrak{n}=\mathfrak{n}^{1} \supset \cdots \supset \mathfrak{n}^{r+1}=0$ be the lower central series of $\mathfrak{n}$, i.e. $\mathfrak{n}^{k+1}=\left[\mathfrak{n}^{k}, \mathfrak{n}\right]$ for $k \geqslant 1$. We will show by induction that

$$
\begin{aligned}
& \mathfrak{n}^{k} \text { is orthogonal to }\left[\mathfrak{n}, \mathfrak{n}_{0}\right] \text { for all } k \geqslant 1, \\
& \mathfrak{n}^{k} \text { is orthogonal to } \mathfrak{n}_{0} \text { for all } k \geqslant 2 \text {. }
\end{aligned}
$$

We then clearly get Proposition 5.5 by taking $k=1$ in (1) and $k=2$ in (2).

Statements (1) and (2) are trivial for $k=r+1$. Now take $j \geqslant 1$ and assume (1) and (2) hold for all $k>j$. Let $Y \in \mathfrak{n}^{j} \cap\left(\mathfrak{n}^{j+1}\right)^{\perp}$ and $X \in \mathfrak{n}_{0}$. Then $[X, Y]$ is in $\mathfrak{n}^{j+1} \cap\left[\mathfrak{n}, \mathfrak{n}_{0}\right]=0$ by (1). The curvature being negative, formula 3.4(11) gives $(\operatorname{ad} X)^{t} Y+(\operatorname{ad} Y)^{t} X=0$ since the definition of $Y$ implies $(\operatorname{ad} Y)^{t} Y$ $\in \mathfrak{a}$ while $(\operatorname{ad} X)^{t} X \in \mathfrak{n}$ by Lemma 5.4(i). But (2) implies $(\operatorname{ad} Y)^{t} X \in \mathfrak{a}$ since the range of ad $\left.Y\right|_{\mathfrak{n}}$ is in $\mathfrak{n}^{j+1}$. Therefore we obtain for all $Z \in \mathfrak{n}, 0=$ $\left\langle(\operatorname{ad} X)^{t} Y, Z\right\rangle=\langle Y,[X, Z]\rangle$.

Thus $\mathfrak{n}^{i} \cap\left(\mathfrak{n}^{j+1}\right)^{\perp}$ is orthogonal to $\left[\mathfrak{n}_{0}, \mathfrak{n}\right]$. By the induction hypothesis $\mathfrak{n}^{j+1}$ is orthogonal to $\left[\mathfrak{n}_{0}, \mathfrak{n}\right]$; hence (1) holds for $k=j$.

Now assume $j \geqslant 2$ and take $X \in \mathfrak{n}^{j} \cap \mathfrak{n}_{0}$. By (1), $[X, Y] \in \mathfrak{n}^{j+1} \cap$ $\left[\mathfrak{n}_{0}, \mathfrak{n}\right]=0$ for all $Y \in \mathfrak{n}$. Hence $X$ is in the center of $\mathfrak{n}$, so that the range of $(\operatorname{ad} X)^{t}$ is in a. In particular by Lemma 5.4(i), (ad $\left.X\right)^{t} X=0$. Using again formula 3.4(11) we conclude that $(\operatorname{ad} X)^{t} Y+(\operatorname{ad} Y)^{t} X=0$ and hence $\left\langle(\operatorname{ad} Y)^{t} X, Z\right\rangle=0$ for all $Z \in \mathfrak{n}, Y \in \mathfrak{n}$. Thus $X$ is orthogonal to $[\mathfrak{n}, \mathfrak{n}]=\mathfrak{n}^{2}$. Since $X \in \mathfrak{n}^{i} \subset \mathfrak{n}^{2}$ we get $X=0$, and hence $\mathfrak{n}^{j} \cap \mathfrak{n}_{0}=0$. Being $a$-invariant, $\mathfrak{n}^{i}$ is a sum of generalized root spaces $n_{\alpha, \beta}$ for which $\alpha$ must be nonzero. Hence, in view of 5.3(ii), $\mathfrak{n}^{j}$ is orthogonal to $n_{0}$, which proves (2) for $k=j$. Q.E.D.

5.6. Proposition. Let 8 be a solvable algebra endowed with a scalar product. Assume 8 has negative curvature. Then (using the notations of 5.1, 5.3) there is an element $\rho$ in $a^{*}$ such that $\langle\alpha, \rho\rangle$ is strictly positive for all $\alpha \in \Delta$.

Proof. As in Proposition 5.5 we use the lower central series $\left\{\mathfrak{n}^{k}: 1 \leqslant\right.$ $k \leqslant r\}$ of $n$. Set $\Delta_{r+1}=\varnothing$ and $\rho_{r+1}=0$. For $k \geqslant 1$, define $\Delta_{k}$ and $\rho_{k}$ 
inductively by $\Delta_{k}=\left\{\alpha \in \Delta: \mathfrak{n}_{\alpha, \beta} \cap \mathfrak{n}^{k} \neq 0\right.$ for some $\beta$, and $\left\langle\alpha, \rho_{l}\right\rangle=0$ for all $l \geqslant k+1\}, \rho_{k}=\Sigma_{\alpha \in \Delta_{k}} \alpha$. We make the following inductive hypothesis: if $\alpha \in$ $\Delta^{k}, \gamma \in \Delta$, and $\left\langle\gamma, \rho_{l}\right\rangle=0$ for all $l \geqslant k+1$, then $\langle\alpha, \gamma\rangle \geqslant 0$ and $\left[\mathfrak{n}_{\alpha, \beta} \cap \mathfrak{n}^{k}, \mathfrak{n}_{\boldsymbol{\gamma}, \delta}\right]$ $=0$ for all $\beta, \delta$.

For $k=r$, this hypothesis is valid by Lemma 5.4(iii) and the fact that $\mathfrak{n}^{r}$ is central in $\mathfrak{n}$. Now, let $j \geqslant 1$ and suppose the induction hypothesis holds for all $k \geqslant j+1$. Fix $\alpha \in \Delta_{j}, \gamma \in \Delta$ with $\left\langle\gamma, \rho_{l}\right\rangle=0$ for all $l \geqslant j+1$ and take $X \in$ $\mathfrak{n}_{\alpha, \beta} \cap \mathfrak{n}^{j}, Y \in \mathfrak{n}_{\gamma, \delta}$. By the Jacobi identity, for all complex roots $\lambda, \mu \in\left(\mathfrak{a}^{*}\right)^{\mathrm{C}}$, $\left[\mathfrak{n}_{\lambda}^{\mathrm{C}}, \mathfrak{n}_{\mu}^{\mathrm{C}}\right] \subset \mathfrak{n}_{\lambda+\mu}^{\mathrm{C}}$, which implies, by construction of the $\mathfrak{n}_{\alpha, \beta}$ (see 5.1), that $[X, Y] \in\left(\mathfrak{n}_{\alpha+\gamma, \beta+\delta}+\mathfrak{n}_{\alpha+\gamma, \beta-\delta}\right) \cap \mathfrak{n}^{j+1}$. Suppose $[X, Y] \neq 0$. Then $\alpha+\gamma \neq$ 0 by Proposition 5.5, and $\alpha+\gamma$ is in $\Delta_{j+1}$. By the induction hypothesis, $\langle\alpha+$ $\gamma, \mu\rangle \geqslant 0$ for all $\mu \in \Delta_{j+1}$ and thus we have the contradictory inequality $0=$ $\left\langle\rho_{j+1}, \alpha+\gamma\right\rangle \geqslant|\alpha+\gamma|^{2}>0$. We conclude that $[X, Y]$ must be zero. Now let $W \in \mathfrak{n}_{\xi, \eta}$. By 5.3(iii), if $\alpha$ and $\xi$ are independent, $\left\langle(\operatorname{ad} X)^{t} X, W\right\rangle=\langle X,[X, W]\rangle=$ 0 . If $\alpha$ and $\xi$ are dependent, $[X, W]=0$ by the above argument where $\xi$ plays the part of $\boldsymbol{\gamma}$. Hence $(\operatorname{ad} X)^{t} X$ is in a. Then for $Y \in \mathfrak{n}_{\gamma, \delta}$, we conclude from Lemma 5.4(ii) that

$$
\left\langle(\operatorname{ad} X)^{t} X,(\operatorname{ad} Y)^{t} Y\right\rangle=\langle\alpha, \gamma\rangle|\alpha|^{-2}|\gamma|^{-2}\left\langle\left[H_{\alpha}, X\right], X X\left[H_{\gamma}, Y\right] Y\right\rangle .
$$

By formula 3.4(11), negativity of the curvature implies that this expression is $\geqslant 0$. Selecting $X$ and $Y$ as in Lemma 5.4(iii), we get $\langle\alpha, \gamma\rangle \geqslant 0$ which gives the induction hypothesis for $k=j$.

Now, for any $\alpha \in \Delta$, let $k(\alpha)$ be the unique index for which $\left\langle\alpha, \rho_{k(\alpha)}\right\rangle \neq 0$ and $\left\langle\alpha, \rho_{k}\right\rangle \neq 0$ for all $k \geqslant k(\alpha)+1$. Note that the above argument implies $k(\alpha) \geqslant j$ whenever $\mathfrak{n}_{\alpha, \beta} \cap \mathfrak{n}^{j} \neq 0$ for some $\beta$. Moreover $\langle\alpha, \gamma\rangle \geqslant 0$ for all $\gamma \in$ $\Delta_{k(\alpha)}$, so $\left\langle\alpha, \rho_{k(\alpha)}\right\rangle$ is strictly positive. Take $c$ between 0 and 1 such that

$$
c \sum_{j=1}^{r}\left|\left\langle\alpha, \rho_{j}\right\rangle\right|\left\langle\left\langle\alpha, \rho_{k(\alpha)}\right\rangle \quad \text { for all } \alpha \in \Delta\right.
$$

and define $\rho=\rho_{r}+c \rho_{r-1}+\cdots+c^{r-1} \rho_{1}$.

We have, for all $\alpha \in \Delta$

$$
\langle\alpha, \rho\rangle=c^{r-k(\alpha)}\left(\left\langle\alpha, \rho_{k(\alpha)}\right\rangle+\cdots+c^{k(\alpha)-1}\left\langle\alpha, \rho_{1}\right\rangle\right)
$$

so that

$$
\langle\alpha, \rho\rangle \geqslant c^{r-k(\alpha)}\left(\left\langle\alpha, \rho_{k(\alpha)}\right\rangle-c \sum_{j=1}^{k(\alpha)-1}\left\langle\alpha, \rho_{j}\right\rangle\right)>0 \text {. Q.E.D. }
$$

5.7. REMARKs. Let $\mathfrak{b}=\mathfrak{a} \oplus \mathfrak{n}$ be a Lie algebra endowed with an inner product for which it has negative curvature. Consider two roots $(\alpha+i \beta)$ and 
$(\gamma+i \delta)$ of $\mathfrak{a}$ in $\mathfrak{n}$ with nonzero real parts. If $\alpha$ and $\gamma$ are proportional, we must have $\gamma=c \alpha$ for a strictly positive constant $c$ in view of Proposition 5.6. This has useful consequences concerning the Lie algebra structure of $\mathfrak{n}$.

By the Jacobi identity, for all $\alpha, \beta, \gamma, \delta$

$$
\left[\mathfrak{n}_{\alpha, \beta}, \mathfrak{n}_{\gamma, \delta}\right] \subset \mathfrak{n}_{\alpha+\gamma, \beta+\delta}+\mathfrak{n}_{\alpha+\gamma, \beta-\delta} .
$$

On the other hand, our definition $\mathfrak{n}_{j}=\Sigma_{c \neq 0, \beta \in a^{*}} \mathfrak{n}_{c \alpha_{j}, \beta}$ now implies $\mathfrak{n}_{j}=$ $\Sigma_{c>0, \beta \in \mathfrak{a}^{*}} \mathfrak{n}_{c \alpha_{j}, \beta}$. In particular the subspaces $\mathfrak{n}_{j}, 0 \leqslant j \leqslant m$, are subalgebras of $\mathfrak{n}$.

One sees similarly that for $j \neq k,\left[\mathfrak{n}_{j}, \mathfrak{n}_{k}\right]$ and $\left[\mathfrak{n}_{j},\left[\mathfrak{n}_{j}, \mathfrak{n}_{k}\right]\right]$ are orthogonal to $\mathfrak{n}_{j} \oplus \mathfrak{n}_{k}$, since $c \alpha_{j}+d \alpha_{k}$ cannot be proportional to $\alpha_{j}$ or $\alpha_{k}$ for $c, d>0$.

5.8. We are now going to use the structural information gathered about solvable Lie algebras with negative curvature to establish more explicit formulas for the covariant differentiation operator $\nabla$ and the curvature tensor $R$. These computations do not use any assumption on the negativity of sectional curvature, but only structural constraints on the pair $(\xi,\langle\cdot, \cdot\rangle)$ which we list as follows:

(i) 8 is a solvable Lie algebra, endowed with an inner product. The orthogonal complement $\mathfrak{a}$ of $\mathfrak{n}=[\boldsymbol{z}, \boldsymbol{z}]$ is abelian.

(ii) There are elements $\alpha_{j}, 0 \leqslant j \leqslant m$, in $a^{*}$ with $\alpha_{0}=0$, and $\alpha_{1}, \ldots, \alpha_{m}$ pairwise independent such that $\mathfrak{n}=\bigoplus_{0 \leqslant j \leqslant m} \mathfrak{n}_{j}$ where, for each $j, \mathfrak{n}_{j}$ is the sum of all generalized root spaces $\mathfrak{n}_{\alpha, \beta}$ for which $\alpha$ is a positive multiple of $\alpha_{j}$. bra of $\mathfrak{n}$.

(iii) $\mathfrak{n}_{0}$ is contained in the center of $\mathfrak{n}$ and $\mathfrak{n}_{+}=\bigoplus_{1<j \leqslant m} \mathfrak{n}_{j}$ is a subalge-

(iv) There exists a linear operator $E$ on $\mathfrak{n}$ which leaves each subspace $\mathfrak{n}_{j}$ invariant and commutes with ad $H$ for all $H \in \mathfrak{a}$. Moreover, for $P_{j}$ the orthogonal projection of $\mathfrak{n}$ on $\mathfrak{n}_{j}$ and $E_{j}=E \circ P_{j}$, ad $H-\Sigma_{1 \leqslant j \leqslant m} \alpha_{j}(H) E_{j}$ is skew-symmetric for all $H \in \mathfrak{a}$.

We introduce some additional notations. For $j=1, \ldots, m$, write $H_{j}$ in place of $H_{\alpha_{j}}$; thus, $\left\langle H_{j}, H\right\rangle=\alpha_{j}(H)$ for all $H \in \mathfrak{a}$. The conventions of $\S 3.2$ are systematically used to identify $\Lambda^{2} z$ with the Lie algebra of skew-symmetric operators on 8. For $X \in \mathfrak{n}$, we define $D_{X} \in \Lambda^{2} \mathfrak{n} \subset \Lambda^{2}$ as the component in $\Lambda^{2} \mathfrak{n}$ of $\nabla_{X} \in \Lambda^{2}$, i.e. $D_{X}=P \circ \nabla_{X} \circ P$ where $P$ is the orthogonal projection of 8 onto $\mathfrak{n}$. It follows from 3.1(1) that for $X, Y \in \mathfrak{n}$,

$$
D_{X} Y=1 / 2\left\{[X, Y]-\left(\left.\operatorname{ad} X\right|_{\mathfrak{n}}\right)^{t} Y-\left(\left.\operatorname{ad} Y\right|_{\mathfrak{n}}\right)^{t} X\right\} .
$$

5.9. Lemma. Let be a solvable Lie algebra endowed with an inner product and suppose the structural conditions $5.8(\mathrm{i})$-(iv) are satisfied. Let $\nabla$ be the covariant differentiation operator and $R$ the curvature tensor on 8. Recall that $\operatorname{Re}(A)$ and $\operatorname{Im}(A)$ stand for the symmetric and skew-symmetric parts of an operator $A$. We then have 


$$
\nabla_{H}=\operatorname{Im}(\operatorname{ad} H) \text { for } H \in \dot{a} ;
$$

$$
\nabla_{X}=\sum_{j=1}^{m} \operatorname{Re}\left(E_{j}\right) X \wedge H+D_{X} \quad \text { for } X \in \mathfrak{n}
$$

$$
R(X \wedge H)=\sum_{j=1}^{m} \alpha_{j}(H) N\left(E_{j}\right) X \wedge H_{j}+\left[D_{X}, D_{H}\right]-D_{[X, H]}
$$

for $X \in \mathfrak{n}, H \in \mathfrak{a}$, where $N\left(E_{j}\right)=\left(\operatorname{Re} E_{j}\right)^{2}+\left[\operatorname{Re} E_{j}, \operatorname{Im} E_{j}\right]$;

$$
R(X \wedge Y)=\sum_{j=1}^{m}\left(D_{X} \operatorname{Re}\left(E_{j}\right) Y-D_{Y} \operatorname{Re}\left(E_{j}\right) X-\operatorname{Re}\left(E_{j}\right)[X, Y]\right) \wedge H_{j}
$$

$$
\begin{array}{r}
+\sum_{j, k=1}^{m}\left\langle\alpha_{j}, \alpha_{k}\right\rangle \operatorname{Re}\left(E_{j}\right) X \wedge \operatorname{Re}\left(E_{k}\right) Y+\left[D_{X}, D_{Y}\right]-D_{[X, Y]} \\
\text { for } X, Y \in \mathfrak{n} ;
\end{array}
$$

$\langle R(X \wedge Y), X \wedge K-Y \wedge H\rangle=\left\langle 3[X, Y] / 2+(\operatorname{ad} X)^{t} X / 2, h Y-k X\right\rangle$

$$
\begin{aligned}
+ & \langle X,[X, k Y]+[k X, Y]\rangle \\
& -\langle Y,[h X, Y]+[X, h Y]\rangle
\end{aligned}
$$

for all $X, Y \in \mathfrak{n}, H, K \in \mathfrak{a}$, where $h=\operatorname{Re}(\operatorname{ad} H), k=\operatorname{Re}(\operatorname{ad} K)$.

Proof. The orthogonality of $\mathfrak{a}$ and $\mathfrak{n}$ yields $(\operatorname{ad} X)^{t} H=0$ for $X \in \Delta$, $H \in$ a. Hence 3.2(7) implies

$$
\nabla_{H}=1 / 2\left(\operatorname{ad} H-(\operatorname{ad} H)^{t}\right)=\operatorname{Im}(\operatorname{ad} H)
$$

for $H \in \mathfrak{a}$ and

$$
\nabla_{X} H=-\operatorname{Re}(\operatorname{ad} H) X
$$

for $H \in a, X \in \mathfrak{n}$. In view of 5.8(iv) and 3.2(4), (10) may be rewritten as

$$
\nabla_{X} H=-\sum_{j=1}^{m} \alpha_{j}(H) \operatorname{Re}\left(E_{j}\right) X=\sum_{j=1}^{m}\left(\operatorname{Re}\left(E_{j}\right) X \wedge H_{j}\right) H .
$$

Using the definition of $D_{X}$ given in 5.8 and skew-symmetry of $\nabla_{X}$, this implies (6).

It is easily deduced from 5.8(i)-(iv) that, for $H \in \mathfrak{a}$, the skew-symmetric operator $J(H)=\operatorname{ad} H-\Sigma_{j=1}^{m} \alpha_{j}(H) E_{j}$ commutes with $E_{j},\left(E_{j}\right)^{t}$, ad $K$, and $(\operatorname{ad} K)^{t}$ for all $j=1, \ldots, m$ and all $K \in \mathfrak{a}$. In particular, the operators $\operatorname{Im}(\operatorname{ad} H), H \in$ $a$, form a commutative family.

To obtain formulas (7) and (8), one substitutes the expressions for $\nabla_{\boldsymbol{X}}$ and $\nabla_{H}$ given by (5) and (6) into the expression 3.1(2) for the curvature. We omit 
the details, but point out that the computation makes use of the commutative properties just observed for the operators $J(H)$ as well as the rule (easily deduced from the conventions in 3.2) that $[A, X \wedge Y]=A X \wedge Y+X \wedge A Y$ for $X, Y \in$ 8 and $A$ a skew-symmetric operator on 8 .

To prove formula (9), we first use 3.1(2) and 3.2(6) to obtain for $X, Y \in \mathfrak{n}$, $K \in \mathfrak{a}$,

$$
\langle R(X \wedge Y), K \wedge X\rangle=\left\langle\left(\nabla_{X} \nabla_{Y}-\nabla_{Y} \nabla_{X}-\nabla_{[X, Y]}\right) K, X\right\rangle .
$$

By skew-symmetry of $\nabla_{X}$ and (10), we may write, with the notation $\operatorname{Re}(\operatorname{ad} K)=k$,

$$
\left\langle\nabla_{X} \nabla_{Y} K, X\right\rangle=-\left\langle\nabla_{Y} K, \nabla_{X} X\right\rangle=\left\langle k Y, \nabla_{X} X\right\rangle
$$

and use 3.2(7) to evaluate $\nabla_{X} X$. Similar computations transform (11) into

$$
\begin{aligned}
\langle R(X \wedge Y), K \wedge X\rangle= & -\left\langle k Y,(\operatorname{ad} X)^{t} X\right\rangle+\left\langle k X,(\operatorname{ad} X)^{t} Y+(\operatorname{ad} Y)^{t} X\right\rangle / 2 \\
& +3\langle[X, Y], k X\rangle / 2 .
\end{aligned}
$$

Using the formula analogous to (12) for $\langle R(X \wedge Y), H \wedge Y\rangle$, (9) is deduced by elementary manipulations. Q.E.D.

5.10. LEMMA. Let be a solvable Lie algebra endowed with an inner product and suppose the structural conditions 5.8(i)-(iv) are satisfied.

(i) Call $a_{0}$ the space of elements $H \in$ a such that $\alpha(H)=0$ for any root $\alpha+i \beta$ of $a$ in $n$. The restriction of the curvature tensor $R$ of to $a \wedge a$ and $\left(a_{0}+\mathfrak{n}_{0}\right) \wedge z$ is identically zero.

(ii) Call $L$ the orthogonal complement of $\sum_{j=1}^{m} \mathfrak{n}_{j} \wedge H_{j}$ in $\mathfrak{n} \wedge a$. Denote a typical element in $\mathfrak{n} \wedge$ a by $L=\Sigma_{t} X_{t} \wedge K_{t}$ with $X_{t} \in \mathfrak{n}, K_{t} \in \mathfrak{a}$. For $P_{j}$, $1 \leqslant j \leqslant m$, as in 5.8(iv), the following three properties are equivalent:

$L$ is in $L$;

$\Sigma_{t} \alpha_{j}\left(K_{t}\right) P_{j} X_{t}=0$ for $j=1,2, \ldots, m$;

$\Sigma_{t} \operatorname{Re}\left(\operatorname{ad} K_{t}\right) X_{t}=0$.

(iii) $R(L)$ and $L$ are orthogonal in $\Lambda^{2}$.

Proof. Formulas (5) and 3.1(2) imply $R(a \wedge a)=\{0\}$ since, as seen in Lemma 5.9, the operators $\operatorname{Im}(\operatorname{ad} H), H \in \mathfrak{a}$, commute with one another.

For $X \in \mathfrak{n}_{0}, 5.8$ (iii) implies that $D_{X}=0$. Using (6), we obtain

$$
\nabla_{X}=0 \quad \text { for } X \in \mathfrak{n}_{0} .
$$

Since $\mathfrak{n}_{0}$ is an ideal in $8,(13)$ and 3.1(2) imply $R\left(\mathfrak{n}_{0} \wedge \boldsymbol{b}\right)=\{0\}$.

For $H \in \mathfrak{n}_{0}, \operatorname{Re}(\operatorname{ad} H)=0$ by $5.8(\mathrm{iv})$ and hence $\nabla_{H}=\operatorname{Im}(\operatorname{ad} H)=\operatorname{ad} H$ is a skew-symmetric derivation of 8 . But for any skew-symmetric derivation $L$ of $\mathbf{6}$, a tedious but routine computation based on $3.2(7)$ shows that 


$$
\left[L, \nabla_{X}\right]=\nabla_{L X} \quad \text { for all } X \in 8 .
$$

In particular, for $H \in \dot{a}_{0}, 3.1(2)$ gives $R(H \wedge X)=0$ for all $X \in$ 8. This completes the proof of 5.10(i).

Let $L$ be as in 5.10(ii) and $j \geqslant 1$. With the help of formula 3.2(5), the orthogonality of $\Sigma_{t} X_{t} \wedge K_{t}$ and $n_{j} \wedge H_{j}$ is seen to be equivalent to $\Sigma_{t} \alpha_{j}\left(K_{t}\right) P_{j}\left(X_{t}\right)$ $=0$. Since $N(E)=\operatorname{Re}(E)^{2}+[\operatorname{Re}(E), \operatorname{Im}(E)] \geqslant 0$, we see that the kernel of $\operatorname{Re}(E)$ is contained in the kernel of $N(E)$ and hence is invariant under $\operatorname{Im}(E)$. It follows from 5.3(iv) that the kernel of $\operatorname{Re}(E) h_{n_{j}}$ is a-invariant and spanned by root vectors corresponding to roots with zero real parts. Since $\mathfrak{n}_{j} \cap \mathfrak{n}_{0}=\{0\}$, we conclude that $\left.\operatorname{Re}(E)\right|_{\mathfrak{n}_{j}}=\left.\operatorname{Re}\left(E_{j}\right)\right|_{\mathfrak{n}_{j}}$ is invertible. But 5.3(iv) implies that $\alpha_{j}\left(K_{t}\right) \operatorname{Re}\left(E_{j}\right)=P_{j} \operatorname{Re}\left(\operatorname{ad} K_{t}\right)$ for all $t$. Hence $\Sigma_{t} \alpha_{j}\left(K_{t}\right) \nabla_{j}\left(X_{t}\right)=0$ if and only if $P_{j} \Sigma_{t} \operatorname{Re}\left(\operatorname{ad} K_{t}\right) X_{t}=0$ and this proves (ii).

To check (iii), we simply note that formula (7) implies that $R(\mathfrak{n} \wedge \mathfrak{n}$ ) is contained in $\Sigma_{j} \mathfrak{n}_{j} \wedge H_{j}+\mathfrak{n} \wedge \mathfrak{n}$ and this space is orthogonal to $L$ in $\Lambda^{2}$. Q.E.D.

5.11. THEOREM. Let 8 be a solvable Lie algebra endowed with an inner product. Assume that 8 has negative curvature, so that $=\mathfrak{a} \oplus \mathfrak{n}$ with a abelian, $\mathfrak{n}=[8,8]$. Then $\mathfrak{n}$ is the orthogonal direct sum of a-invariant subspaces $\mathfrak{n}^{0}$ and $\mathfrak{n}^{1}$ having the following properties.

(i) $\mathfrak{n}^{0}=\Sigma \mathfrak{n}_{\alpha, \beta}^{0}$ where the sum is taken over all roots $\alpha+i \beta$ of $a$ in $\mathfrak{n}$ and $\mathfrak{n}_{\alpha, \beta}^{0}=\left\{X \in \mathfrak{n}_{\alpha, \beta}:\right.$ ad $\left.X\right|_{\mathfrak{n}_{\gamma, \delta}}=0$ whenever $\alpha$ and $\gamma$ are independent $\}$.

(ii) ad $\left.H\right|_{n^{1}}$ is normal for all $H \in \mathfrak{a}$.

Proof. Throughout the proof we shall use the notations and results of 5.1 and 5.3. We begin by establishing several technical formulas.

Let $L \subset \mathfrak{n} \wedge \mathfrak{a}$ be as in Lemma 5.10. We show first that whenever $X, Y \in$ $\mathfrak{n}, H, K \in \mathfrak{a}$, and $X \wedge K-Y \wedge H \in L$, then

$$
\langle X,[\operatorname{Re}(\operatorname{ad} K) X, Y]+[X, \operatorname{Re}(\operatorname{ad} K) Y]\rangle
$$

$$
=\langle Y,[\operatorname{Re}(\operatorname{ad} H) X, Y]+[X, \operatorname{Re}(\operatorname{ad} H) Y]\rangle .
$$

Indeed, for any real scalar $t$, let $Z_{t}=(X+t H) \wedge(Y+t K)=X \wedge Y+$ $t(X \wedge K-Y \wedge H)$. Since the sectional curvature on is negative, $\left\langle R\left(Z_{t}\right), Z_{t}\right\rangle \geqslant$ 0 for all $t$. By Lemma 5.10(iii) and the fact that $R$ is a symmetric linear operator on $\Lambda^{2} 8$, we conclude that

$$
0 \leqslant\left\langle R\left(Z_{t}\right), Z_{t}\right\rangle=\langle R(X \wedge Y), X \wedge Y\rangle+2 t\langle R(X \wedge Y), X \wedge K-Y \wedge H\rangle
$$

for all $t$. Obviously, this condition can be satisfied only if

$$
\langle R(X \wedge Y), X \wedge K-Y \wedge H\rangle=0
$$


From Lemma 5.10(ii), we have $\operatorname{Re}(\operatorname{ad} K) X=\operatorname{Re}(\operatorname{ad} H) Y$. Using this and formula (9) to compute the left-hand side of (16), we obtain (15),

Now suppose $j, k, l$ are distinct indices $(1 \leqslant j, k, l \leqslant m)$ and $c, d$ positive scalars such that $\alpha_{l}$ is a positive multiple of $c \alpha_{j}+d \alpha_{k}$. Fix $K, H \in a$ and write $a_{p}=\alpha_{p}(K), b_{p}=\alpha_{p}(H)$ for $p=1,2, \ldots, m$. Since $\alpha_{j}$ and $\alpha_{k}$ are independent, we may assume $b_{j} b_{k} b_{l}\left(a_{j} b_{k}-a_{k} b_{j}\right) \neq 0$. Let $X=X_{j}+X_{k}+X_{l}$ be an arbitrary vector in $\mathfrak{n}_{j} \oplus \mathfrak{n}_{k} \oplus \mathfrak{n}_{l}$ and define $Y=Y_{j}+Y_{k}+Y_{l}$ by

$$
b_{p} Y_{p}=a_{p} X_{p} \quad \text { for } p=j, k, l .
$$

By Lemma 5.10(ii), $X \wedge K-Y \wedge H \in L$ and hence (15) holds. We now expand (15) and note that $\operatorname{Re}(K)=\Sigma_{p} a_{p} \operatorname{Re}\left(E_{p}\right), \operatorname{Re}(H)=\Sigma b_{p}\left(\operatorname{Re} E_{p}\right)$; the result is a collection of terms each of which lies in $\left\langle\mathfrak{n}_{p},\left[\mathfrak{n}_{q}, \mathfrak{n}_{r}\right]\right\rangle$ for some choice of indices $p, q, r$ in $\{j, k, l\}$. The remarks in 5.7 show that such terms are automatically zero except when either $p=q=r$ or $p=l,\{q, r\}=\{j, k\}$. However, the terms corresponding to $p=q=r$ cancel out in view of (17). After easy manipulations with the remaining terms and systematic use of (17), we obtain

$$
\left\langle X_{l}, d\left[\operatorname{Re}\left(E_{j}\right) X_{j}, X_{k}\right]-c\left[X_{j}, \operatorname{Re}\left(E_{k}\right) X_{k}\right]\right\rangle=0 .
$$

Obviously, (18) remains valid under complexification, i.e. under replacement of $X_{j}, X_{k}, X_{l}$ by arbitrary vectors in $\mathfrak{n}_{j}^{\mathbf{C}}, \mathfrak{n}_{k}^{\mathbf{C}}, \mathfrak{n}_{l}^{\mathbf{C}}$, respectively.

We now define, for $j=1,2, \ldots, m$, a subspace $\mathfrak{n}_{j}^{0}$ of $\mathfrak{n}_{j}^{\mathbf{C}}$ by

$$
\mathfrak{n}_{j}^{0}=\left\{X \in \mathfrak{n}_{j}^{\mathrm{C}}:[X, Y]=0 \text { for all } Y \in \mathfrak{n}_{k}^{\mathrm{C}} \text { and } 1 \leqslant k \neq j \leqslant m\right\}
$$

and call $\mathfrak{n}_{j}^{1}$ the orthogonal complement of $n_{j}^{0}$ in $n_{j}^{c}$. Since $n_{j}^{C}$ and $n_{k}^{C}$ are $a$ invariant, it follows from the Jacobi identity that $\mathfrak{n}_{j}^{0}$ is $a$-invariant. By construction, $\left[\mathfrak{n}_{j}, \mathfrak{n}_{k}\right.$ ] is contained in the sum of all subspaces $\mathfrak{n}_{l}$ for which $\alpha_{l}$ is a positive multiple of $c \alpha_{j}+d \alpha_{k}$ with $c, d>0$. Hence, (18) implies that $\mathfrak{n}_{j}^{0}$ is $\operatorname{Re}(E)$-invariant. We now claim that $\mathfrak{n}_{j}^{0}$ is $E$-invariant as well and hence $E^{*}$-invariant. To see this, fix $j \neq k$ and note that $X \in \mathfrak{n}_{j}^{0}$ if and only if ad $X\left(\mathfrak{n}_{\mu}^{\mathbf{C}}\right)=\{0\}$ for all complex roots $\mu$ with real part a positive multiple of $\alpha_{k}$. Writing $X=\Sigma X_{\lambda}$ with $X_{\lambda} \in \mathfrak{n}_{\lambda}^{\mathrm{C}}$ and the sum taken over all complex roots $\lambda$ with real part a positive multiple of $\alpha_{j}$, it follows that $X \in \mathfrak{n}_{j}^{0}$ if and only if $X_{\lambda} \in \mathfrak{n}_{j}^{0}$ for all $\lambda$. Now fix $X \in \mathfrak{n}_{j}^{0} \cap \mathfrak{n}_{\lambda}^{\mathrm{C}}$ and $Y \in \mathfrak{n}_{\mu}^{\mathrm{C}} \subset \mathfrak{n}_{k}^{\mathrm{C}}$. Then there are linear functionals $\beta$ and $\gamma$ in $a^{*}$ such that $\left.\left(\operatorname{ad} H-\alpha_{j}(H) E\right)\right|_{n} \mathrm{c}=i \beta(H) I,\left.\left(\operatorname{ad} H-\alpha_{k}(H) E\right)\right|_{n_{\mu} \mathrm{c}}=i \gamma(H) I$ for all $H \in \cdot$ a. Using the Jacobi identity, this implies

$$
\begin{aligned}
0 & =(\operatorname{ad} H)[X, Y] \\
& =\left[\alpha_{j}(H) E X, Y\right]+\left[X, \alpha_{k}(H) E Y\right]+i(\beta(H)+\gamma(H))[X, Y] \\
& =\alpha_{j}(H)[E X, Y] .
\end{aligned}
$$

By the above argument, we can conclude from (19) that $E X \in \mathfrak{n}_{j}^{0}$ and hence $\mathfrak{n}_{j}^{0}$ 
is $E$-invariant. Finally, $\mathfrak{n}_{j}^{0}$ is invariant under $(\mathrm{ad} H)^{*}$ for all $H \in \mathfrak{a}$ since $5.3(\mathrm{v})$ implies that on $\mathfrak{n}_{j}^{\mathbf{C}},(\operatorname{ad} H)^{*}=\alpha_{j}(H) E^{*}-\left(\operatorname{ad} H-\alpha_{j}(H) E\right)$.

We now turn to $\mathfrak{n}_{j}^{1}$, the orthogonal complement of $\mathfrak{n}_{j}^{0}$ in $\mathfrak{n}_{j}$. Since $\mathfrak{n}_{j}^{0}$ is invariant under $E, E^{*}$, and, for all $H \in \mathfrak{a},(\operatorname{ad} H)$ and $(\operatorname{ad} H)^{*}$, the same is true of $\mathfrak{n}_{j}^{1}$. The remainder of the proof of the theorem is devoted to showing that $a$ acts normally on $\mathfrak{n}_{j}^{1}$. Once this is accomplished, the subspaces $\mathfrak{n}^{0}=\mathfrak{n}_{0} \oplus \mathfrak{n} \cap$ $\left(\bigoplus_{1<j<m} \mathfrak{n}_{j}^{0}\right)$ and $\mathfrak{n}^{1}=\mathfrak{n} \cap\left(\bigoplus_{1<j \leqslant m} \mathfrak{n}_{j}^{1}\right)$ clearly have the properties (i) and (ii) stated in the theorem.

For each root $\nu$, denote by $Q_{\nu}$ the natural (direct sum) projection of $\mathfrak{n}^{\mathrm{c}}=$ $\Sigma_{\lambda} \mathfrak{n}_{\lambda}^{\mathbf{C}}$ onto $\mathfrak{n}_{\nu}^{\mathbf{c}}$. The $\boldsymbol{a}$-invariance of $\mathfrak{n}_{j}^{1}$ implies the invariance of $\mathfrak{n}_{j}^{1}$ under $Q_{\nu}$. Now take $j \neq k$, roots $\lambda=c \alpha_{j}+i \beta, \mu=d \alpha_{k}+i \gamma$ with $c, d>0$, and $X \in \mathfrak{n}_{\lambda}^{\mathbf{C}} \cap$ $\mathfrak{n}_{j}^{1}, Y \in \mathfrak{n}_{\mu}^{\mathrm{C}} \cap \mathfrak{n}_{k}^{1}$. Applying (18) with $X=X_{j}, Y=X_{k}$ and $X_{l}$ arbitrary, we conclude that

$$
d[\operatorname{Re}(E) X, Y]=c[X, \operatorname{Re}(E) Y]
$$

and hence

$$
d \sum_{\nu}\left[Q_{\nu} \operatorname{Re}(E) X, Y\right]=c \sum_{\rho}\left[X, Q_{\rho} \operatorname{Re}(E) Y\right] .
$$

By 5.3(ii), $Q_{\nu} \operatorname{Re}(E) X$ is zero unless $\nu$ is of the form $z \alpha_{j}+i \beta$ with $z \in \mathrm{C}$ and then $\left[Q_{\nu} \operatorname{Re}(E) X, Y\right] \in \mathfrak{n}_{\sigma}^{\mathrm{C}}$ with $\sigma=\nu+\mu=z \alpha_{j}+d \alpha_{k}+i(\beta+\gamma)$. Similarly, $Q_{\rho} \operatorname{Re}(E) Y$ is zero unless $\rho$ is of the form $z^{\prime} \alpha_{k}+i \gamma$ and then $\left[X, Q_{\rho} \operatorname{Re}(E) Y\right] \in$ $\mathfrak{n}_{\sigma^{\prime}}^{\mathbf{C}^{\prime}}$ with $\sigma^{\prime}=\lambda+\rho=c \alpha_{j}+z^{\prime} \alpha_{k}+i(\beta+\gamma)$. Since $\alpha_{j}$ and $\alpha_{k}$ are independent, $\sigma=\sigma^{\prime}$ if and only if $\nu=\lambda$ and $\rho=\mu$. Hence (21) implies

$$
\left[Q_{\nu} \operatorname{Re}(E) X, Y\right]=0=\left[X, Q_{\rho} \operatorname{Re}(E) Y\right]
$$

whenever $\nu \neq \lambda, \rho \neq \mu$. By holding $\lambda, X$ fixed and allowing $\mu, Y$ to vary, we deduce from (22) that $Q_{\nu} \operatorname{Re}(E) X \in \mathfrak{n}_{j}^{0}$ for $\nu \neq \lambda$. Since $\mathfrak{n}_{j}^{1}$ is invariant under $\operatorname{Re}(E)$ and $Q_{\nu}$, it follows that $Q_{\nu} \operatorname{Re}(E) X \in \mathfrak{n}_{j}^{1} \cap \mathfrak{n}_{j}^{0}=\{0\}$ for $\nu \neq \lambda$ and hence $\operatorname{Re}(E) X=Q_{\lambda} \operatorname{Re}(E) X \in \mathfrak{n}_{j}^{1} \cap \mathfrak{n}_{\lambda}^{\mathrm{C}}$. Thus we have shown that $\operatorname{Re}(E)$ leaves $\mathfrak{n}_{j}^{1} \cap$ $\mathfrak{n}_{\lambda}^{C}$ invariant for all roots $\lambda$. These spaces are $E$-invariant from above and hence $E^{*}$-invariant as well.

Let $E=S+N$ be the Jordan decomposition of $E$, i.e. $S$ and $N$ are polynomials in $E$ with $S$ semisimple and $N$ nilpotent (Humphreys [6, p. 17]). For any root $\lambda$ with real part a multiple of $\alpha_{j}$, denote $\mathfrak{n}_{j}^{1} \cap \mathfrak{n}_{\lambda}^{C}$ by $\mathfrak{v}_{\lambda}$. Since $\mathfrak{b}_{\lambda}$ is invariant under $E$ and $E^{*}$, it is invariant under $S, S^{*}, N$ and $N^{*}$. For $\lambda=c \alpha_{j}+$ $i \beta, 5.3(\mathrm{iv})$ implies that the restriction of $S$ to $\mathfrak{n}_{\lambda}^{\mathbf{C}}$ is a scalar operator $\left(c+i c^{\prime}\right) I$ for some $c^{\prime} \in \mathbf{R}$. Since $\mathfrak{D}_{\lambda}$ is both $S$ - and $S^{*}$-invariant, $\left.S^{*}\right|_{\mathfrak{D}_{\lambda}}=\left(\left.S\right|_{\mathfrak{b}_{\lambda}}\right)^{*}=$ $\left(c-i c^{\prime}\right) I$. Consequently, the restrictions of $S$ and $S^{*}$ to $\mathfrak{n}_{j}^{1}$ commute, $\left.S\right|_{\mathfrak{n}_{1} 1}$ is normal, and $\left.\operatorname{Re}(S)\right|_{0_{\lambda}}=c I$ for $\lambda=c \alpha_{j}+i \beta$. Upon replacement of $\operatorname{Re}(E)$ by $\operatorname{Re}(S)+\operatorname{Re}(N),(20)$ yields 


$$
d[\operatorname{Re}(N) X, Y]=c[X, \operatorname{Re}(N) Y]
$$

for $X \in \mathfrak{v}_{c \alpha_{j}+i \beta}, Y \in \mathfrak{v}_{d \alpha_{k}+i \gamma}$. Now let $N(H)$ be the nilpotent part of the derivation ad $H$ of $\mathfrak{n}^{\mathrm{C}}$. Then $N(H)$ is again a derivation $[6$, p. 18] and, in view of 5.3(iv), is related to $N$ by $\left.N(H)\right|_{\mathfrak{n} C}=\left.\alpha_{j}(H) N\right|_{n_{j}}$. For $X \in \mathfrak{n}_{c \alpha_{j}+i \beta}^{\mathrm{C}}, Y \in \mathfrak{n}_{d \alpha_{k}+i \gamma}^{\mathrm{C}}$, and $l$ the unique index for which $b \alpha_{l}=c \alpha_{j}+d \alpha_{k}$ for some $b>0$, it follows that

$$
\alpha_{l}(H) N[X, Y]=\alpha_{j}(H)[N X, Y]+\alpha_{k}(H)[X, N Y] .
$$

Again using linear independence of $\alpha_{j}$ and $\alpha_{k}$, we conclude that

$$
c d N[X, Y] / b=d[N X, Y]=c[X, N Y] .
$$

It is convenient to define a new nilpotent operator $M$ on $\bigoplus_{1 \leqslant j \leqslant m} \mathfrak{n}_{j}^{\mathrm{C}}$ by requiring that $\left.M\right|_{\mathfrak{n}_{\lambda}}=N /\left.c\right|_{\mathfrak{n}_{\lambda}}$ for $\lambda=c \alpha_{j}+i \beta$. From (23) and (24), we obtain

$$
\left[M^{*} X, Y\right]=\left[X, M^{*} Y\right], \quad M[X, Y]=[M X, Y]=[X, M Y]
$$

for all $X \in \mathfrak{n}_{j}^{1}, Y \in \mathfrak{n}_{k}^{1}, j \neq k$. We also know that if $X \in \mathfrak{n}_{j}^{1}$ and $[M X, Y]=0$ for all $Y \in \bigoplus_{k \neq j} \mathfrak{n}_{k}^{1}$, then $M X \in \mathfrak{n}_{j}^{1} \cap \mathfrak{n}_{j}^{0}=\{0\}$. We claim that these conditions force $\left.M\right|_{\mathfrak{n}_{j}^{1}}$ to be zero. To see this, write $\mathfrak{v}$ and $\mathfrak{u}$ in place of $\mathfrak{n}_{j}^{1}$ and $\bigoplus_{k \neq j} \mathfrak{n}_{k}^{1}$, denote by $P$ and $Q$ the restrictions of $M$ to $\boldsymbol{D}$ and $\mathfrak{u}$, and consider the orthogonal decompositions $\mathfrak{v}=\mathfrak{v}_{0} \oplus \mathfrak{v}_{1}, \mathfrak{u}=\mathfrak{u}_{0} \oplus \mathfrak{u}_{1}$ where $\mathfrak{v}_{0}$ (respectively, $\mathfrak{u}_{0}$ ) is the kernel of $P$ (respectively, $Q$ ). Thus $\mathfrak{v}_{1}=P^{*} \mathfrak{v}, \mathfrak{u}_{1}=Q^{*} \mathfrak{u}$. First assume $P^{2}=0$. Then $P * b_{1}=0$. From (25) we obtain

$$
[P \mathfrak{v}, \mathfrak{u}]=\left[P \mathfrak{v}_{1}, \mathfrak{u}_{0}\right]+\left[P \mathfrak{v}_{1}, Q^{*} \mathfrak{u}\right]=\left[\mathfrak{v}_{1}, Q \mathfrak{u}_{0}\right]+M\left[P^{*} \mathfrak{v}_{1}, \mathfrak{u}\right]=\{0\}
$$

and thus $P=0$. In general, $P^{2 k}=0$ for some $k \geqslant 1$. Since the conditions in (25) remain valid under replacement of $M$ by $M^{k}$, the case just considered implies $P^{k}=0$ and hence by a trivial induction, $P=0$.

We now have $\left.N\right|_{\mathfrak{n}_{j}}=0$ and hence $\left.E\right|_{\mathfrak{n}_{j}^{1}}=\left.S\right|_{\mathfrak{n}_{j} 1}$ is normal. By 5.3(iv), ad $\left.H\right|_{\mathfrak{n}_{j}}$ is normal for all $H \in \mathfrak{a}$. Q.E.D.

\section{A class of solvable algebras.}

6.1. Notations. As seen in $\S 5$, the existence of a metric with negative curvature on a Lie algebra imposes strong structural restrictions on itself. We gather these constraints in Definition 6.2 after establishing a few notational conventions.

Let 8 be a real solvable Lie algebra and $\mathfrak{n}=[8,8]$. If there exists in 8 subalgebra a complementary to $\mathfrak{n}, \boldsymbol{a}$ must clearly be abelian, and we follow the conventions of 5.1 regarding roots $\lambda=\alpha+i \beta \in\left(\mathfrak{a}^{*}\right)^{\mathrm{C}}$ of $\mathfrak{a}$ in $\mathfrak{n}^{\mathrm{C}}$, root spaces $\mathfrak{n}_{\lambda}^{\mathbf{C}}$, and generalized root spaces $\mathfrak{n}_{\alpha, \beta}$. 
In such a situation, we set

$$
\begin{aligned}
\Delta & =\left\{\alpha \in a^{*}: \alpha \neq 0, \text { and } \alpha+i \beta \text { is a root for some } \beta \in a^{*}\right\}, \\
\mathfrak{n}_{+} & =\sum_{\alpha \in \Delta, \beta \in a^{*}} n_{\alpha, \beta}, \\
n_{0} & =\sum_{\beta \in a^{*}} \mathfrak{n}_{0, \beta}, \\
n_{\alpha, \beta}^{0} & =\left\{X \in \mathfrak{n}_{\alpha, \beta}:\left[X, \mathfrak{n}_{\gamma, \delta}\right]=0 \text { whenever } \gamma \text { and } \alpha \text { are independent }\right\}, \\
n^{0} & =\sum_{\alpha, \beta \in a^{*}} \mathfrak{n}_{\alpha, \beta}^{0} .
\end{aligned}
$$

6.2. Definition. A Lie algebra 8 is said to be of negative curvature type (abbreviated, is an $N C$ algebra) if 8 is solvable and has the following properties (using the notations of 6.1):

(i) there exists in a (necessarily abelian) subalgebra a complementary to $\mathfrak{n}=[\mathbf{8}, \mathbf{8}]$;

(ii) for each root $\lambda=\alpha+i \beta$ of $a$ in $\mathfrak{n}^{\mathbf{C}}$, ad $\left.H\right|_{n} \mathbf{c}$ is semisimple if $H \in \mathfrak{a}$ and $\alpha(H)=0$ (in particular, ad $\left.H\right|_{n} c=0$ if $H \in a$ and $\lambda(H)=0$ );

(iii) $\Delta$ lies in an open half-space of $a^{*}$ (i.e. there exists $H_{0} \in \mathfrak{a}$ such that $\alpha\left(H_{0}\right)>0$ whenever $\alpha+i \beta$ is a root and $\left.\alpha \neq 0\right)$;

(iv) $\mathfrak{n}_{0}$ lies in the center of $\mathfrak{n}$;

(v) for all $\alpha, \beta \in a^{*}, \mathfrak{n}_{\alpha, \beta}^{0}$ is $a$-invariant and admits in $\mathfrak{n}_{\alpha, \beta}$ an $a$-invariant complement $\mathfrak{n}_{\alpha, \beta}^{1}$ upon which the action of $a$ is semisimple.

6.3. REMARKS. If 8 is a Lie algebra endowed with an inner product and if 8 has negative curvature, then the results of $\S 5$ show that 8 is an $N C$ algebra. The converse will be proved in $\$ 7$.

A class of examples of $N C$ algebras is provided by those solvable Lie algebras which arise in the Iwasawa decomposition of semisimple Lie algebras, i.e. the Lie algebras of groups acting simply transitively by isometries on a noncompact symmetric space. In this setting, conditions (i) and (iii) are automatic from the construction of such algebras. The roots are real valued and the action of $\boldsymbol{a}$ is semisimple, so conditions (ii), (iv) and (v) are trivially satisfied.

The $N C$ algebras for which $\mathfrak{n}$ is abelian are easily classified and constructed since in that case (iv) and (v) are trivially satisfied.

A particular type of $N C$ algebra was introduced by Heintze [4] who studied solvmanifolds with strictly negative sectional curvature. They correspond to the case $\operatorname{dim} a=1, \mathfrak{n}_{0}=0$. In this case, (iv) and (v) are trivially satisfied, while (ii) and (iii) simply mean that all eigenvalues of ad $H_{0}$, for some nonzero $H_{0} \in \mathfrak{a}$, have strictly positive real parts. We will come back to this example in 7.8. In order to obtain a classification of $N C$ algebras, one must in particular solve the 
apparently difficult problem of determining which nilpotent Lie algebras admit a derivation all of whose eigenvalues have nonnegative real parts.

The class of $N C$ algebras is clearly closed under Lie algebra direct sums and passage to quotients by ideals. However, the class is not closed under restriction to an ideal. In particular, the only nilpotent $N C$ algebras are abelian by (ii) and (iv).

Given an $N C$ algebra $=\mathfrak{a}+\mathfrak{n}$ as in 6.2 , it is easy to see that $n_{+}$is an ideal of 8 and that the center of is a subspace of $a$. Moreover, when $\lambda=$ $\alpha+i \beta$ is a root, Definition 6.2 implies that ad $\left.H\right|_{\mathfrak{n}} \mathbf{c}=\alpha(H) F_{\lambda}+i \beta(H) I$ where $F_{\lambda}$ is a fixed operator on $\mathfrak{n}_{\lambda}^{\mathbf{C}}$.

We shall now show that Definition 6.2 does not depend on the choice of a particular subalgebra a of complementary to $\mathfrak{n}$. We begin with a definition and a lemma modeled on analogous concepts for the case of Iwasawa subalgebras of a semisimple subalgebra (e.g., see Wallach [12, p. 174]).

6.4. Definition. Let 8 be an $N C$ algebra. An element $Z \in \&$ is said to be regular if $\operatorname{ad}_{\mathfrak{n}} Z$ is nonsingular.

Note that for $\mathfrak{a}$ as in $6.2, H+X$ is regular whenever $X \in \mathfrak{n}, H \in \mathfrak{a}$, and $\lambda(H) \neq 0$ for all roots $\lambda$. Since the collection of roots is finite, the set of regular elements is dense in 8 . We let Aut(8) be the group of automorphisms of 8 and $N$ the subgroup of Aut(8) consisting of elements of the form $\nu=e^{\operatorname{ad} X}$ for $X \in$ $\mathfrak{n}$. Thus, $N$ is a connected nilpotent Lie group with Lie algebra ad $(\mathfrak{n})$. Note that $\mathrm{ad}_{\mathfrak{b}} \mathfrak{n}$ is isomorphic to $\mathfrak{n}$ since Definition 6.2 implies that the center of 8 is contained in $\boldsymbol{a}$.

6.5. Lemma. Let $=\mathfrak{a}+\mathfrak{n}$ be a solvable Lie algebra satisfying 6.2(i)(iv). Let $H$ be a regular element in a. Then the mapping $\Phi: N \rightarrow \xi$ defined for $\nu \in N$ by $\Phi(\nu)=\nu \cdot H-H$ is a diffeomorphism from $N$ onto $\mathfrak{n}$.

Proof. Since $H$ normalizes $\mathfrak{n}, e^{\operatorname{ad} X}(H)-H$ is in $\mathfrak{n}$ for all $X \in \mathfrak{n}$ and hence $\Phi$ is a $C^{\infty}$-mapping from $N$ onto $\mathfrak{n}$. For any $\nu \in N$, we identify $\mathfrak{n}$ with the tangent space $T_{\nu}(N)$ in the obvious fashion. As usual, we also identify $\mathfrak{n}$ with the tangent space $T_{\Phi(\nu)}(\mathfrak{n})$. Then $(d \Phi)_{\nu}: T_{\nu}(N) \rightarrow T_{\Phi(\nu)}(\mathfrak{n})$ is simply the mapping $X \rightarrow[X, H]=-\operatorname{ad} H(X)$. Since $H$ is a regular element, this means that $\Phi$ is everywhere regular as a $C^{\infty}$-mapping of manifolds. Therefore, $\Phi$ is a local diffeomorphism.

From 6.2(iv), it follows that $N$ is the direct product of $N_{0}=\left\{e^{\mathrm{ad} X}: X \in\right.$ $\left.\mathfrak{n}_{0}\right\}$ and $N_{+}=\left\{e^{\operatorname{ad} X}: X \in \mathfrak{n}_{+}\right\}$. Clearly, $\Phi\left(N_{+}\right) \subset \mathfrak{n}_{+}$and $\Phi\left(e^{\text {ad } X} \nu\right)=[X, H]$ $+\Phi(v)$ for $X \in \mathfrak{n}_{0}, \nu \in N_{+}$. Consequently, there is no loss of generality in assuming that $\mathfrak{n}=\mathfrak{n}_{+}$. By 6.2(iii), we may find an element $H_{0} \in \mathfrak{a}$ with

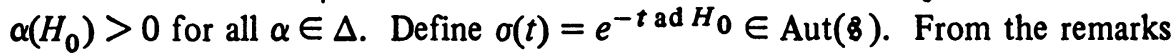
in 6.3, for each root $\lambda=\alpha+i \beta,\left.\sigma(t)\right|_{n_{\lambda}}=e^{-i t \beta\left(H_{0}\right)} e^{-t \alpha\left(H_{0}\right) F}$ for $F$ a fixed 
unipotent operator on $\mathfrak{n}_{\lambda}^{\mathbf{C}}$. This implies $\lim _{t \rightarrow \infty} \sigma(t) X=0$ for all $X \in \mathfrak{n}$. For $X \in \mathfrak{n}$ and $\nu=e^{\operatorname{ad} X} \in N$, it follows that $\lim _{t \rightarrow \infty} \sigma(t) \nu \sigma(t)^{-1}=\lim _{t \rightarrow \infty} e^{\operatorname{ad} \sigma(t) X}=$ identity element of $\operatorname{Aut}(z)$. Moreover, $\sigma(t) H=H$ since $a$ is abelian, and this implies

$$
\Phi\left(\sigma(t) \nu \sigma(t)^{-1}\right)=\sigma(t) \Phi(\nu)
$$

Because $(d \Phi)_{e}$ is nonsingular, there exists a neighborhood $U$ of the identity in $N$ which is mapped diffeomorphically onto a neighborhood $V$ of 0 in $\mathfrak{n}$. Given any $X \in \mathfrak{n}, \sigma(t) X \in V$ for sufficiently large $t$. Since $\sigma(t)$ normalizes $N$, (1) implies that $X \in \sigma(t)^{-1} \Phi(N)=\Phi(N)$. Similarly, for $\nu_{1}$ and $v_{2}$ arbitrary in $N, \sigma(t) \nu_{1} \sigma(t)^{-1}$ and $\sigma(t) \nu_{2} \sigma(t)^{-1}$ belong to $V$ for sufficiently large $t$. From (1) it follows that $\Phi\left(\nu_{1}\right)=\Phi\left(\nu_{2}\right)$ implies $\nu_{1}=\nu_{2}$. Q.E.D.

6.6. Proposition. Let 8 be an NC algebra with $\mathfrak{n}=[8,8]$. Let $\mathfrak{a}$ be an arbitrary subalgebra of which is complementary to $\mathfrak{n}$. Then $\mathfrak{a}$ is abelian an properties 6.2(ii)-(iv) hold. Moreover, any other subalgebra $a^{\prime}$ of 8 complementary to $\mathfrak{n}$ is the image of a by an automorphism $\sigma$ of 8. One may take $\sigma=e^{\mathrm{ad} X}$ where $X \in \mathfrak{n}$ is uniquely determined by a and $\mathfrak{a}^{\prime}$.

Proof. By definition, we may find an abelian subalgebra $a$ of for which properties $6.2(\mathrm{i})-(\mathrm{v})$ hold. Let $\boldsymbol{a}^{\prime}$ be any other subalgebra of complementary to $\mathfrak{n}$. Since properties $6.2(\mathrm{i})-(\mathrm{v})$ are preserved under automorphisms, to prove 6.6 , it is enough to exhibit a unique element $\nu \in N$ (see Lemma 6.5 for notations) such that $v(\mathfrak{a})=\mathfrak{a}^{\prime}$. Let $H$ be a regular element in $\mathfrak{a}$. The direct sum decompositions $\mathbf{z}=\mathfrak{a}+\mathfrak{n}=\mathfrak{a}^{\prime}+\mathfrak{n}$ guarantee the existence of an element $H^{\prime} \in \dot{a}^{\prime}$ such that $H-H^{\prime} \in \mathfrak{n}$. By Lemma 6.5, we may find $\nu \in N$ such that $\nu(H)=H^{\prime}$. Since $H$ is regular, a equals $z(H)$, the centralizer of $H$ in 8 . But $\nu$ is an automorphism and hence $v(z(H))=z\left(H^{\prime}\right)$, the centralizer of $H^{\prime}$ in 8 . Since $a^{\prime}$ is necessarily abelian, $a^{\prime} \subset z\left(H^{\prime}\right)$. However, $\operatorname{dim} a=\operatorname{dim} \mathfrak{a}^{\prime}=\operatorname{codim} \mathfrak{n}$, and it follows that $\dot{a}^{\prime}=\delta\left(H^{\prime}\right)=v(a)$. The uniqueness of $\nu$ follows from Lemma 6.5. Q.E.D.

7. Construction of metrics with negative curvature.

7.1. Our goal now is to prove that any $N C$ algebra carries at least one (and, in fact, many) metrics with negative curvature. We start with a metric on $\boldsymbol{z}=\mathfrak{a}+\mathfrak{n}$ for which the operators ad $H, H \in \mathfrak{a}$, resemble almost normal operators. Skillful linear "deformation" transforms this metric into a metric with negative curvature. These deformations are equivalent to modifications of the Lie algebra structure of $\&$ which make $\mathfrak{n}$ "almost" abelian.

7.2. Defintion. Let be an $N C$ algebra. An inner product on 8 is said to be admissible if the following conditions are met (using the notations of 6.1). 
(i) The orthogonal complement $\mathfrak{a}$ of $\mathfrak{n}$ in $\boldsymbol{z}$ is a (necessarily abelian) subalgebra.

(ii) Under the canonical extension (see 4.1) of the inner product from $\mathfrak{n}$ to $\mathfrak{n}^{\mathbf{C}}$, complex root spaces $\mathfrak{n}_{\lambda}^{\mathbf{C}}$ and $\mathfrak{n}_{\mu}^{\mathbf{C}}$ of $\boldsymbol{a}$ are orthogonal whenever $\lambda$ and $\mu$ are inequivalent roots (see 5.1).

(iii) The orthogonal complement $\mathfrak{n}^{1}$ of $\mathfrak{n}^{0}=\Sigma_{\alpha, \beta \in a^{*}} \mathfrak{n}_{\alpha, \beta}^{0}$ in $\mathfrak{n}$ (see 6.1) is a-invariant, and ad $\left.H\right|_{\mathfrak{n} 1}$ is normal for all $H \in \mathfrak{a}$.

The results of $\S 5$ show that if 8 is a Lie algebra endowed with a metric having negative curvature, 8 must be an $N C$ algebra and the inner product on 8 must be admissible.

Now if 8 is an $N C$ algebra, admissible inner products on 8 obviously exist in view of Definition 6.2 (take, for instance, the subspaces $\mathfrak{n}_{\lambda}^{\mathbf{C}}$ to be pairwise orthogonal and use 6.2 (v) to guarantee that 7.2(iii) holds).

Whenever we have an $N C$ algebra $\delta=a \oplus \mathfrak{n}$ endowed with an admissible inner product, we select elements $\alpha_{j}, 0 \leqslant j \leqslant m$, in $a^{*}$ with $\alpha_{0}=0$ and with the property that for any root $\alpha+i \beta$ there exists a unique index $j$ such that $\alpha=c \alpha_{j}$ for some $c>0$. For $j=0,1, \ldots, m$, we define $H_{j}$ as the unique vector in a such that $\left\langle H_{j}, H\right\rangle=\alpha_{j}(H)$ for all $H \in \mathfrak{a}$ and set $\mathfrak{n}_{j}=\Sigma_{c>0, \beta \in a^{*}} \mathfrak{n}_{c \alpha_{j}, \beta}$. Hence $\mathfrak{n}=\bigoplus_{0 \leqslant j \leqslant m} \mathfrak{n}_{j}$. Using Definitions 6.2 and 7.2 instead of Theorem 4.8, Corollary 4.9 , and Theorem 5.2 , we proceed along essentially the same steps as in $\S 5.3$ to obtain a finer decomposition

$$
\mathfrak{n}^{\mathrm{C}}=\bigoplus_{p} \mathfrak{W}_{p}
$$

corresponding to equivalence classes of roots, and to construct a linear operator $E$ on $\mathfrak{n}^{\mathbf{C}}$ which is equal to zero on $\mathfrak{n}_{0}$, commutes with the adjoint action of $\mathfrak{a}$, leaves invariant the subspaces $\mathfrak{n}_{\alpha, \beta}, \mathfrak{n}_{\lambda}^{\mathrm{C}}, \mathfrak{n}_{j}$, and $\mathfrak{Q}_{p}$, and satisfies 5.3(i)-(v). In particular, for $E_{j}$ the composition of $E$ with the orthogonal projection from $\mathfrak{n}$ to $\mathfrak{n}_{j}$, the operators

$$
J(H)=\operatorname{ad} H-\sum_{1 \leqslant j \leqslant m} \alpha_{j}(H) E_{j}
$$

are skew-symmetric for $H \in \mathfrak{a}$. By construction, for $\mathfrak{\bigotimes}_{\boldsymbol{p}} \subset \mathfrak{n}_{j}^{\mathbf{C}}$, the restriction of $J(H)$ to $\mathfrak{\bigotimes}_{p}$ is a scalar operator. Since the complexifications of $\mathfrak{n}^{0}$ and $\mathfrak{n}^{1}$ are the direct sums of their intersections with the various subspaces $\bigvee_{p}$ and are $a$ invariant, it follows that $\mathfrak{n}^{0}$ and $\mathfrak{n}^{1}$ are $E$-invariant.

We now settle the case where $\mathfrak{n}$ is abelian which will play the role of a "limiting situation" in our approach for the general case.

7.3. Proposition. Let be an NC algebra endowed with an admissible inner product. Assume that $\mathfrak{n}=[\overline{8}, 8]$ is abelian. Then 8 has negative curvature 
if and only if the curvature tensor $R$ is positive when viewed as a symmetric operator on $\Lambda^{2}$ \&. In general, $R$ vanishes on the orthogonal complement of $\mathfrak{n}_{+} \wedge \mathfrak{n}_{+}+\Sigma_{1 \leqslant j \leqslant m} \mathfrak{n}_{j} \wedge H$ in $\Lambda^{2}$. . Using the notations of 4.1 and $7.2, R$ is positive (respectively, positive definite) on $\mathfrak{n}_{+} \wedge \mathfrak{n}_{+}+\Sigma_{1 \leqslant j \leqslant m} \mathfrak{n}_{j} \wedge H_{j}$ if and only if

(i) $\operatorname{Re}(E)$ and $N(E)$ are positive (respectively, positive definite);

(ii) for $1 \leqslant j, k \leqslant m,\left\langle H_{j}, H_{k}\right\rangle$ is nonnegative (respectively, strictly positive).

Proof. As seen in 7.2, properties 5.8(i)-(iv) hold. Consequently, we may make use of the results and notations of 5.8,5.9,5.10, and 7.2. Since $\mathfrak{n}$ is abelian, 5.8(4) implies that $D_{X}=0$ for $X \in \mathfrak{n}$. Therefore, formulas 5.9(7) and $5.9(8)$ simplify to

$$
\begin{aligned}
& R(X \wedge H)=\sum_{1<j<m} \alpha_{j}(H) N\left(E_{j}\right) X \wedge H_{j}, \\
& R(X \wedge Y)=\sum_{1<j \leqslant m}\left\langle H_{j}, H_{k}\right\rangle \operatorname{Re}\left(E_{j}\right) X \wedge \operatorname{Re}\left(E_{k}\right) Y,
\end{aligned}
$$

for $X, Y \in \mathfrak{n}, H \in \mathfrak{a}$.

By (3), when $j \geqslant 1$ and $X \in \mathfrak{n}_{j}$ is an eigenvector of $N(E)$ for the eigenvalue $a, X \wedge H_{j}$ is an eigenvector of $R$ for the eigenvalue $a\left|H_{j}\right|^{2}$. By (4), when $1 \leqslant j$, $k \leqslant m$ and $X \in \mathfrak{n}_{j}, Y \in \mathfrak{n}_{k}$ are eigenvectors of $\operatorname{Re}(E)$ for the eigenvalues $b, c$, then $X \wedge Y$ is an eigenvector of $R$ for the eigenvalue $b c\left\langle H_{j}, H_{k}\right\rangle$. The collection of eigenvectors for $R$ just described span $\mathfrak{n}_{+} \wedge \mathfrak{n}_{+}+\Sigma_{1 \leqslant j \leqslant m} \mathfrak{n}_{j} \wedge H_{j}$. Using the notations of 5.10, the orthogonal complement of this collection is $\left(a_{0}+\mathfrak{n}_{0}\right)$ $\wedge+\mathfrak{a} \wedge \mathfrak{a}+L$. But Lemma 5.10 and formula (3) imply that $R$ vanishes on this subspace. Consequently, it is possible to find an orthonormal basis of $\Lambda^{2} 8$ with the property that each element in the basis is an elementary tensor (i.e. may be written in the form $V \wedge W$ for $V, W \in 8$ ) and an eigenvector of $R$. It follows that the symmetric operator $R$ on $\Lambda^{2} Z$ is positive if and only if $\langle R(Z), Z\rangle \geqslant 0$ for every elementary tensor $Z \in \Lambda^{2} \&$, i.e. if and only if $z$ has negative curvature. The above list of eigenvalues for $R$ shows immediately that conditions (i) and (ii) imply $R \geqslant 0$.

Conversely, if $R \geqslant 0$, we have $a \geqslant 0$ whenever $a$ is an eigenvalue of $N(E)$ and $b c \geqslant 0$ whenever $b$ and $c$ are eigenvalues of $\operatorname{Re}\left(\left.E\right|_{n_{j}}\right)$ for $j=1,2, \ldots, m$. Thus $N(E) \geqslant 0$ and all eigenvalues of $\operatorname{Re}\left(\left.E\right|_{\mathfrak{n}_{j}}\right)$ have the same sign. However, all eigenvalues of $\left.E\right|_{\mathfrak{n}_{j}}$ have strictly positive real parts for $j=1,2, \ldots, m$, and this implies

$$
\operatorname{trace} \operatorname{Re}\left(\left.E\right|_{\mathfrak{n}_{j}}\right)=\operatorname{trace}\left(\left.E\right|_{\mathfrak{n}_{j}}\right)>0 .
$$

Since $E_{\mathfrak{m}_{0}}=0$ by convention, $\operatorname{Re}(E)$ must be positive. By (5), for $1 \leqslant j, k \leqslant m$, 
we may select eigenvalues $b$ of $\operatorname{Re}\left(\left.E\right|_{\mathfrak{n}_{j}}\right)$ and $c$ of $\operatorname{Re}\left(\left.E\right|_{\mathfrak{n}_{k}}\right)$ with $b c>0$. From above, $b c\left\langle H_{j}, H_{k}\right\rangle \geqslant 0$ and we deduce that $\left\langle H_{j}, H_{k}\right\rangle \geqslant 0$.

The statements in Proposition 7.3 regarding positive definite operators follow trivially. Q.E.D.

7.4. Lemma. Let $A$ be a linear operator on a finite dimensional real Hilbert space $\$$. Let $\mathcal{C}$ be a set of linear operators on $\$$ such that $C \in \mathcal{C}$ implies $C^{t} \in \mathcal{C}$ and $C A=A C$. Then there exist linear operators $T_{1}, T_{2}, \ldots$ on $\$$ which are invertible, selfadjoint, commute with the elements of $C$, and have the property that $\lim _{n \rightarrow \infty} T_{n} A T_{n}^{-1}$ exists and is normal.

Proof. We first handle some special cases. If $A$ is a scalar operator (in particular, if $\mathfrak{W}$ is one-dimensional), then $A$ is already normal and there is nothing to prove. Now suppose $A$ is semisimple and has only the eigenvalues $\lambda$ and $\bar{\lambda}$ for some complex scalar $\lambda$. Thus $\mathfrak{Q}^{\mathrm{C}}$, the complexification of $\mathfrak{Q}$, is the direct sum of the eigenspaces $\bigvee_{\lambda}^{C}$ and $\bigvee_{\lambda}^{C}$. Denote by $\langle\cdot, \cdot\rangle$ the canonical extension to $\bigvee^{C}$ of the inner product on $\mathfrak{Q}$. Let $\{\cdot, \cdot\}$ be the unique Hermitian form on $\mathfrak{\$}^{\mathbf{C}}$ satisfying the conditions

$$
\{v, w\}= \begin{cases}0 & \text { if } v \in \bigvee_{\lambda}^{\mathrm{C}}, w \in \bigvee_{\bar{\lambda}}^{\mathrm{C}}, \\ \langle v, w\rangle & \text { if } v, w \in \bigvee_{\lambda}^{\mathrm{C}} \text { or } v, w \in \bigvee_{\bar{\lambda}}^{\mathrm{C}}\end{cases}
$$

Trivially $\{\cdot, \cdot\}$ is an inner product and hence there exists a positive definite operator $P$ on $\mathfrak{Q}^{\mathbf{C}}$ such that $\{v, w\}=\langle P v, w\rangle$ for all $v, w \in \mathfrak{\Phi}$. Note that relative to $\{\cdot, \cdot\}, A^{\mathrm{C}}$ is normal since its eigenspaces are orthogonal. An easy computation shows that this implies $P^{1 / 2} A^{\mathrm{C}}\left(P^{1 / 2}\right)^{-1}$ is normal relative to $\langle\cdot, \cdot\rangle$ where, as usual, $P^{1 / 2}$ denotes the unique positive definite operator whose square is $P$. We now claim that $P^{1 / 2}$ is the complexification of an operator $T$ (necessarily selfadjoint) on $\mathfrak{Q}$ commuting with the elements of $\mathcal{C}$. Given this claim, we obtain $T A T^{-1}$

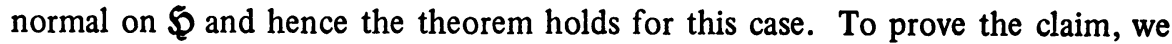
must show that $P^{1 / 2}$ commutes both with the conjugation operator $J$ on $\mathfrak{Q}^{C}$ and the operators obtained by complexification of the elements of $C$. It is enough to show that $P$ commutes with these operators since any operator commuting with $P$ automatically commutes with any function of $P$. For $C \in C$, we continue to write $C$ in place of $C^{\mathbf{C}}$ and note that $C$ and $C^{*}$ leave invariant the eigenspaces $\mathfrak{Q}_{\lambda}^{C}$ and $\mathfrak{Q}_{\lambda} \frac{\mathrm{C}}{\lambda}$. Consequently, if $v$ and $w$ belong to the same eigenspace, the definition of $\{\cdot, \cdot\}$ implies that

$$
\langle P C v, w\rangle=\langle C v, w\rangle=\left\langle v, C^{*} w\right\rangle=\left\langle P v, C^{*} w\right\rangle=\langle C P v, w\rangle .
$$

If $v$ and $w$ belong to different eigenspaces, however, we obtain

$$
\langle P C v, w\rangle=\{C v, w\}=0=\left\{v, C^{*} w\right\}=\left\langle P v, C^{*} w\right\rangle=\langle C P v, w\rangle .
$$


It follows that $P C=C P$. Making use of the fact that $J$ interchanges the eigenspaces of $A$ and has the property $\langle J v, w\rangle=\langle\overline{v, J w}\rangle$ for all $v, w \in \mathfrak{Q}^{\mathrm{C}}$, a similar computation shows that $P J=J P$.

For the general case, we use induction on $\operatorname{dim} \$$. We pick any (real or complex)

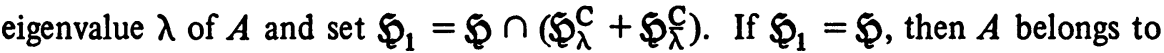
one of the two special operator classes considered above. Otherwise, define $\mathfrak{W}_{2}=\mathfrak{W}_{1}^{\perp}$. Trivially, $\mathfrak{W}_{1}$ is $C$-invariant; since $C$ is closed under the transpose operation, $\mathfrak{Q}_{2}$ is $C$-invariant as well. For $j=1,2$, let $Q_{j}$ be the orthogonal projection of $\mathfrak{Q}$ on $\mathfrak{Q}_{j}, A_{j}=Q_{j} A Q_{j}$, and $C_{j}=\left\{\left.C\right|_{\Phi_{j}}: C \in \mathcal{C}\right\}$. Apply the induction hypothesis to $\left(A_{j}, C_{j}, \mathfrak{Q}_{j}\right)$ and thereby obtain sequences $T_{n, j}(n \geqslant 1)$ of selfadjoint invertible operators on $\$_{j}$ which commute with $C_{j}$ and have the property that $B_{j}=\lim _{n \rightarrow+\infty} T_{n, j} A_{j} T_{n, j}^{-1}$ exists and is normal.

Let $a_{n}, n \geqslant 1$, be any sequence of strictly positive real numbers such that

$$
\lim _{n \rightarrow+\infty} a_{n}\left\|T_{n, 1}\right\|\left\|\left(T_{n, 2}\right)^{-1}\right\|=0 .
$$

Define $T_{n}=a_{n} T_{n, 1} Q_{1}+T_{n, 2} Q_{2}$. It is easily checked that $T_{n}$ is selfadjoint, invertible, commutes with $C$ and $\lim _{n \rightarrow+\infty} T_{n} A T_{n}^{-1}=B_{1} Q_{1}+B_{2} Q_{2}$ is normal. Q.E.D.

7.5. LemMA. Let $(V,\langle\cdot, \cdot\rangle)$ be a finite-dimensional real Hilbert space and $B_{1}: V \times V \rightarrow V$ a Lie algebra structure, i.e. $B_{1}$ is alternating bilinear and satisfies the Jacobi identity. Suppose $T$ is an invertible linear operator on $V$. Let $\left(8_{1},\langle\cdot, \cdot\rangle_{1}\right)$ denote the pair consisting of the Lie algebra $8_{1}$ defined on $V$ by $B_{1}$ and the inner product $\langle\cdot, \cdot\rangle_{1}$ defined by $\langle X, Y\rangle_{1}=\langle T X, T Y\rangle$. Let $\left(8_{2},\langle\cdot, \cdot\rangle_{2}\right)$ denote the pair consisting of the Lie algebra $\delta_{2}$ defined on $V$ by the Lie algebra structure $B_{2}(X, Y)=T B_{1}\left(T^{-1} X, T^{-1} Y\right)$ and the inner product $\langle\cdot, \cdot\rangle_{2}=\langle\cdot, \cdot\rangle$. Then $\left\langle R^{(2)}(T X \wedge T Y), T X \wedge T Y\right\rangle_{2}=\left\langle R^{(1)}(X \wedge Y), X \wedge Y\right\rangle_{1}$ for all $X, Y \in V$, where $R^{(j)}, j=1,2$, is the curvature tensor of $\left(8_{j},\langle\cdot, \cdot\rangle_{j}\right)$.

Proof. The definitions imply that $T: \delta_{1} \rightarrow \delta_{2}$ is both a Lie algebra isomorphism and a vector space isometry. Then formulas 3.1(1) and 3.1(2) imply, for $X, Y \in V$,

$$
\nabla_{T X}^{(2)}=T \nabla_{X}^{(1)} T^{-1}
$$

$$
R^{(2)}(T X \wedge T Y)=T R^{(1)}(X \wedge Y) T^{-1},
$$

where $\nabla^{(j)}$ and $R^{(j)}$ are the covariant differentiation and curvature tensors associated with $\left(8_{j},\langle\cdot, \cdot\rangle_{j}\right)$. The lemma follows from (7) and 3.2(6). Q.E.D.

We are now ready to complete the proof of the main result of this paper, characterizing the Lie algebras which admit metrics with negative curvature. The 
case of strictly negative curvature has already been studied by Heintze [4] and we compare his results to ours in 7.8 below.

7.6. ThEOREM. Let be a Lie algebra. There exists a metric on 8 with negative curvature if and only if is an NC algebra (see Definition 6.2).

7.7. Corollary. Let $S$ be a connected Lie group. There exists on $S$ a left-invariant Riemannian metric with negative sectional curvature if and only if the Lie algebra of $S$ is an NC algebra.

Proof. Corollary 7.7 follows immediately from Theorem 7.6. As we have already pointed out, the results of $\S 5$ prove the "only if" part of Theorem 7.6. To show the converse, we assume that $\delta$ is an $N C$ algebra and select arbitrarily an admissible inner product $\langle\cdot, \cdot\rangle$ on 8 . We use the notations of 6.1 and 7.2 and write $\delta=a+n$. Replacement of the inner product on $\boldsymbol{a}$ by any other inner product still results in an admissible inner product on 8. By 6.2(iii), the real parts of the roots lie in an open half-space of $a^{*}$ and hence we may assume our chosen inner product satisfies $\left\langle H_{j}, H_{k}\right\rangle>0$ for $1 \leqslant j, k \leqslant m$. As seen in 7.2, conditions 5.3(i)-(v) and 5.8(i)-(iv) and all of the results of 5.9 and 5.10 are valid.

Define $\boldsymbol{a}_{0}=\{H \in \mathfrak{a}: \alpha(H)=0$ whenever $\alpha$ is the real part of a root $\}$ and let $a_{+}$be the orthogonal complement of $a_{0}$ in $a$. Let $\boldsymbol{g}_{0}=a_{0} \oplus \mathfrak{n}_{0}$ and $\varepsilon_{+}=a_{+} \oplus n_{+}$. Then $\varepsilon_{0}$ and $z_{+}$are subalgebras of $\varepsilon_{\text {with }}\left[z_{0}, z_{+}\right]=\{0\}$ and $\delta=\delta_{0} \oplus \delta_{+}$. Associated with $\delta$ we have, as usual, the curvature tensor $R$ and the covariant differentiation operators $\nabla_{Z}$ for $Z \in$ 8. From 5.8(4), 5.9(5), and 5.9(6), we see easily that $\nabla_{Z}=0$ for $Z \in \varepsilon_{0}$ while, for $Z \in \delta_{+}, \nabla_{Z}$ is zero on $\delta_{0}$, leaves $\delta_{+}$invariant, and its restriction to $\dot{z}_{+}$agrees with the corresponding covariant differentiation operator on the Lie algebra $\varepsilon_{+}$endowed with the restricted inner product. By $3.1(2)$, it follows that $(z,\langle\cdot, \cdot\rangle)$ has negative curvature if and only if $\left(\varepsilon_{+},\langle\cdot, \cdot\rangle\right)$ has negative curvature. Since our proof will involve modifications of $\langle\cdot, \cdot\rangle$ only on $\dot{z}_{+}$, we may assume from now on that $\boldsymbol{\varepsilon}_{0}=\{0\}$ and hence $\mathfrak{n}=\mathfrak{n}_{+}=\Sigma_{1 \leqslant j \leqslant m} \mathfrak{n}_{j}$. In this situation, the eigenvalues of the operator $E$ on $\mathfrak{n}$ introduced in 7.2 all have strictly positive real parts.

As seen in 7.2 , the subspaces $\mathfrak{W}_{p}, \mathfrak{n}^{0}$, and $\mathfrak{n}^{1}$ are invariant under the operators $E$, ad $H$, and $J(H)$ for $H \in \mathfrak{a}$. From the decomposition $\mathfrak{n}^{\mathrm{C}}=\bigoplus_{p} \mathfrak{\bigotimes}_{p}$, we obtain the decompositions $\mathfrak{n}^{0}=\bigoplus_{p}^{0}$ and $\mathfrak{n}^{1}=\bigoplus_{p}^{1}$ where $\mathfrak{v}_{p}^{0}=\mathfrak{n}^{0} \cap$

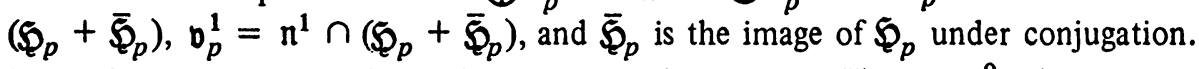
By applying Lemma 7.4 for each index $p$ to the operator $\left.E\right|_{\bullet p}$ on $\nabla_{p}^{0}$ relative to the class $C_{p}=\left\{\left.J(H)\right|_{00}: H \in a\right\}$, we obtain invertible selfadjoint operators $T_{1}, T_{2}, \ldots$ on $\mathfrak{n}$ which commute with $J(H)$ for all $H \in a$, reduce to the identity operator on $\mathfrak{n}^{1}$, leave the subspaces $\boldsymbol{v}_{p}^{0}$ and $\mathfrak{v}_{p}^{1}$ invariant, and have the property that $\lim _{n \rightarrow \infty} T_{n} E T_{n}^{-1}=E_{\infty}$ is a normal operator on $\mathfrak{n}$. Then $T_{n}^{\mathrm{C}}$ leaves invariant 
the complexification $\mathfrak{W}_{p}+\bar{\aleph}_{p}$ of $\mathfrak{v}_{p}^{0}+\mathfrak{v}_{p}^{1}$. Let $\mu_{p}+i v_{p}$ be the element of $\left(a^{*}\right)^{C}$ associated with $\bigvee_{p}$ as in 5.3. By construction, $\mu_{p}-i \nu_{p}$ is the element associated with $\overline{\mathfrak{W}}_{p}, \mathfrak{\Phi}_{p}=\overline{\mathfrak{W}}_{p}$ if $\nu_{p}=0$, and $J(H)=i \nu_{p}(H) I$ on $\mathfrak{\Phi}_{p}$. Since $T_{n}^{\mathrm{C}}$ commutes with $J(H)$ for all $H \in \mathfrak{a}$, it follows that $T_{n}^{\mathbf{C}}$ leaves each subspace $\mathfrak{Q}_{p}$ invariant.

Now for each $n \geqslant 1$, let $a_{n}=n\left\|T_{n}\right\|\left\|T_{n}^{-1}\right\|^{2}$ and define a linear operator $U_{n}$ on \& by

$$
\begin{aligned}
& U_{n}(H)=H \text { for } H \in \mathfrak{a}, \\
& U_{n}(X)=a_{n} T_{n} X \text { for } X \in \mathfrak{n} .
\end{aligned}
$$

It follows easily that

$$
\lim _{n \rightarrow \infty} U_{n}\left[U_{n}^{-1} X, U_{n}^{-1} Y\right]=0
$$

for $X, Y \in \mathfrak{n}$, while for $H \in \mathfrak{n}, X \in \mathfrak{n}_{j}$,

(10) $\lim _{n \rightarrow \infty} U_{n}\left[U_{n}^{-1} H, U_{n}^{-1} X\right]=\lim _{n \rightarrow \infty}\left(T_{n}\right.$ ad $\left.H T_{n}^{-1}\right) X=\left(\alpha_{j}(H) E_{\infty}+J(H)\right) X$.

By construction, $E_{\infty}$ is normal and commutes with $J(H)$ for all $H \in \mathfrak{a}$. Moreover, the eigenvalues of $E_{\infty}$ coincide with those of $E$ and hence have strictly positive real parts. Therefore, $\operatorname{Re} E_{\infty}$ and $N\left(E_{\infty}\right)=\left(\operatorname{Re} E_{\infty}\right)^{2}$ are positive definite operators.

Define Lie algebra structures $B_{n}(n=1,2, \ldots)$ and $B_{\infty}$ on the vector space 8 by

$$
\begin{aligned}
& B_{n}(V, W)=U_{n}\left[U_{n}^{-1} V, U_{n}^{-1} W\right], \\
& B_{\infty}(V, W)=\lim _{n \rightarrow \infty} B_{n}(V, W),
\end{aligned}
$$

for $V, W \in 8$. Denote by $\delta_{n}$ (respectively, $\delta_{\infty}$ ) the Lie algebra with underlying vector space and bracket operation given by $B_{n}$ (respectively, $B_{\infty}$ ). Then $\mathbf{b}_{n}$ and $\delta_{\infty}$ are $N C$ algebras and the admissible inner product $\langle\cdot, \cdot\rangle$ on $\delta$ is an admissible inner product on these new algebras as well. Indeed, note that the properties of the operators $T_{n}$ given above imply that the vector spaces $\mathfrak{n}, \mathfrak{n}^{0}$, $\mathfrak{n}^{1}, \mathfrak{\Phi}_{p}, \mathfrak{a}$, the linear functionals $\alpha_{j}, \mu_{p}, v_{p}$, and the vectors $H_{j} \in \mathfrak{a}$ retain the same interpretations for these new algebras. In particular, as subalgebras of 8 , $\boldsymbol{b}_{n}$, or $\boldsymbol{b}_{\infty}, \mathfrak{a}$ is abelian and $\mathfrak{n}$ is the derived subalgebra. The crucial point, however, is that (9), (10), and (12) imply that as a subalgebra of $\varepsilon_{\infty}, \mathfrak{n}$ is abelian and the adjoint action of $\mathfrak{a}$ on $\mathfrak{n}$ in $\boldsymbol{s}_{\infty}$ is normal.

Denote by $R_{n}$ and $R_{\infty}$ the curvature tensors associated with $\left(\xi_{n},\langle\cdot, \cdot\rangle\right)$ and $\left(\varepsilon_{\infty},\langle\cdot, \cdot\rangle\right)$. Using the properties of $E_{\infty}$ listed above, Proposition 7.3 shows that $R_{\infty}$ is a positive operator on $\Lambda^{2} \delta=\Lambda^{2} \delta_{\infty}$, is positive definite on 
$\mathfrak{n} \wedge \mathfrak{n}+\Sigma_{1 \leqslant j \leqslant m} \mathfrak{n}_{j} \wedge H_{j}$, and vanishes on the orthogonal complement $\mathfrak{a} \wedge \mathfrak{a}+$ $L$ of this subspace in $\Lambda^{2}$ 8. Since $B_{\infty}=\lim _{n \rightarrow \infty} B_{n}$, we have $R_{\infty}=\lim _{n \rightarrow+\infty} R_{n}$, and hence, for sufficiently large $n, R_{n}$ is positive definite on

$$
\left(\mathfrak{n} \wedge \mathfrak{n} \oplus \sum_{1<j \leqslant m} \mathfrak{n}_{j} \wedge H_{j}\right) \text {. }
$$

Unfortunately, although (by Lemma 5.10) $R_{n}$ vanishes on a $\wedge$ a, $R_{n}$ need not vanish on $L$ since $\mathfrak{n}$ as a subalgebra of $\varepsilon_{n}$ is in general not abelian. We shall nevertheless be able to show that for large $n$ the quadratic form $Q_{n}$ defined on $\Lambda^{2} \theta_{n}$ by

$$
Q_{n}(Z)=\left\langle R_{n} Z, Z\right\rangle
$$

for $Z \in \Lambda^{2} z_{n}$ and $1 \leqslant n \leqslant+\infty$ is positive on elementary tensors. This does not imply that $R_{n}$ is a positive operator, but of course guarantees negative sectional curvature on $\left(z_{n},\langle\cdot, \cdot\rangle\right)$.

Let $V=X+H, W=Y+K$ with $X, Y \in \mathfrak{n}$ and $H, K \in \mathfrak{a}$. From Lemma 5.10, and symmetry of $R_{n}$, we get

$$
\begin{aligned}
Q_{n}(V \wedge W)= & Q_{n}(X \wedge K-Y \wedge H) \\
& +2\left\langle R_{n}(X \wedge Y), X \wedge K-Y \wedge H\right\rangle+Q_{n}(X \wedge Y) .
\end{aligned}
$$

Let $P$ be the orthogonal projection of $\mathfrak{n} \wedge \mathfrak{a}$ on $\Sigma_{1<j<m} \mathfrak{n}_{j} \wedge H_{j}$. Since $R_{\infty}$ is a positive operator with kernel $(a \wedge a \oplus L)$, there exists a scalar $a>0$ such that for all $X, Y \in \mathfrak{n}$ and $H, K \in \mathfrak{a}$,

$$
Q_{\infty}(X \wedge Y) \geqslant a|X \wedge Y|^{2}, \quad Q_{\infty}(X \wedge K-Y \wedge H) \geqslant a|P(X \wedge K-Y \wedge H)|^{2} .
$$

Given $\epsilon>0$, it follows that for $n$ sufficiently large,

$$
\begin{aligned}
Q_{n}(X \wedge K-Y \wedge H)+ & Q_{n}(X \wedge Y) \\
& \geqslant(a-\epsilon)\left\{|X \wedge Y|^{2}+|P(X \wedge K-Y \wedge H)|^{2}\right\} .
\end{aligned}
$$

We will show below that for $n$ sufficiently large,

$$
\left.K R_{n}(X \wedge Y), X \wedge K-Y \wedge H\right\rangle|\leqslant \epsilon| X \wedge Y|| P(X \wedge K-Y \wedge H) \mid
$$

holds for all $X, Y \in \mathrm{n}, H, K \in \mathfrak{a}$. Supposing this has been shown, (14)-(16) imply that for $n$ sufficiently large,

$$
\begin{aligned}
Q_{n}(V \wedge W) & \geqslant(a-\epsilon)\left\{|X \wedge Y|^{2}+|P(X \wedge Y-Y \wedge H)|^{2}\right\} \\
& -2 \epsilon|X \wedge Y||P(X \wedge K-Y \wedge H)| .
\end{aligned}
$$

Taking $\epsilon<a / 2$, it follows that there exists $n_{0}>0$ such that $\left(\varepsilon_{n},\langle\cdot, \cdot\rangle\right)$ has negative curvature for all $n \geqslant n_{0}$. Now define new inner products $\langle\cdot, \cdot\rangle_{n}$ on the original Lie algebra 8 by $\langle V, W\rangle_{n}=\left\langle U_{n} V, U_{n} W\right\rangle$ for $V, W \in$. It follows from Lemma 7.5 and the definitions of $B_{n}$ and $\langle\cdot, \cdot\rangle_{n}$ that $\left(\delta,\langle\cdot, \cdot\rangle_{n}\right)$ has negative curvature for all $n \geqslant n_{0}$. 
It remains to verify (16). For this, we shall apply repeatedly the following elementary observation. Suppose

(i) $A_{n}(n \geq 1)$ is a sequence of alternating bilinear maps from $\mathfrak{n} \times \mathfrak{n}$ into n such that $\lim _{n \rightarrow \infty} A_{n}=0$, and

(ii) $C_{n}(n \geqslant 1)$ is a sequence of linear maps from $\mathfrak{n} \wedge \mathfrak{a}$ into $\mathfrak{n}$ such that $\lim _{n \rightarrow \infty} C_{n}$ exists and $C_{n}$ vanishes on the kernel $L$ of $P$ for all $n$.

Then, given $\epsilon>0$, there exists $n_{0}$ such that whenever $n \geqslant n_{0}$, we have

$$
\left.K A_{n}(X, Y), C_{n}(X \wedge K-Y \wedge H)\right\rangle|\leqslant \epsilon| X \wedge Y|| P(X \wedge K-Y \wedge H) \mid
$$

for all $X, Y \in \mathfrak{n}, H, K \in \mathfrak{a}$.

From formula 5.9(9), we may write $\left\langle R_{n}(X \wedge Y), X \wedge K-Y \wedge H\right\rangle=\Xi_{n}+$ $\Theta_{n}$ where

$$
\begin{aligned}
& \Xi_{n}=\left\langle 3 B_{n}(X, Y) / 2+\left(\operatorname{ad}_{n} X\right)^{t} Y / 2-\left(\operatorname{ad}_{n} Y\right)^{t} X / 2, h_{n} Y-k_{n} X\right\rangle, \\
& \Theta_{n}=\left\langle B_{n}\left(X, k_{n} Y\right)+B_{n}\left(k_{n} X, Y\right), X\right\rangle-\left\langle B_{n}\left(X, h_{n} Y\right)-B_{n}\left(h_{n} X, Y\right), Y,\right.
\end{aligned}
$$

and $h_{n}=\operatorname{Re}\left(\operatorname{ad}_{b_{n}} H\right), k_{n}=\operatorname{Re}\left(\operatorname{ad}_{b_{n}} K\right)$.

For the expression $\Xi_{n}$, we let $A_{n}(X, Y)$ be the term appearing on the lefthand side of the inner product and define $C_{n}: \mathfrak{n} \wedge \mathfrak{n} \rightarrow \mathfrak{n}$ by $C_{n}(X \wedge K)=$ $\operatorname{Re}\left(\operatorname{ad}_{b_{n}} K\right) X$. Then (i) is trivially verified since $\mathfrak{n}$ is abelian in $\delta_{\infty}$ while Lemma 5.10 and formula (10) imply that (ii) is verified. We now turn to $\Theta_{n}$. We use the decompositions $X=\Sigma_{\alpha} X_{\alpha}, Y=\Sigma_{\gamma} Y_{\gamma}$ where $X_{\alpha}$ and $Y_{\gamma}$ are the components of $X$ and $Y$ in $\Sigma_{\beta} n_{\alpha, \beta}$ and $\Sigma_{\delta} \mathfrak{n}_{\gamma, \delta}$, respectively. By expanding the expressions involving $B_{n}$ in terms of these components, $\Theta_{n}$ becomes a sum over $\alpha$ and $\gamma$ of expressions involving the inner product of $X$ or $Y$ with elements in $B_{n}\left(\Sigma_{\beta} \mathfrak{n}_{\alpha, \beta}, \Sigma_{\delta} \mathfrak{n}_{\gamma, \delta}\right)$.

We first examine the contribution $\Phi_{n}$ to $\Theta_{n}$ consisting of the partial sum over all $\alpha$ and $\gamma$ which are dependent. For this, we recall that $\alpha$ and $\gamma$ are dependent if and only if there exists an index $j(1 \leqslant j \leqslant m)$ such that $\alpha=c \alpha_{j}, \gamma=d \alpha_{j}$ for some positive scalars $c$ and $d$. Furthermore, $\mathfrak{n}_{j}=\Sigma_{c>0, \beta \in a^{*}} n_{c \alpha_{j}, \beta}$ is a subalgebra of $\boldsymbol{B}_{n}$ orthogonal to $\mathrm{n}_{k}$ for $j \neq k$. Set $D_{j, n}=\operatorname{Re}\left(T_{n} E_{j} T_{n}^{-1}\right), X_{j}=$ $\Sigma_{c>0} X_{c \alpha_{j}}$, and $Y_{j}=\Sigma_{d>0} Y_{d \alpha_{j}}$. Since $h_{n}=\Sigma_{1<j \leqslant m} \alpha_{j}(H) D_{j, n}$ and $k_{n}=$ $\Sigma_{1 \leqslant j \leqslant m} \alpha_{j}(K) D_{j, n}$, we obtain

$$
\Phi_{n}=\sum_{1 \leqslant j \leqslant m}\left\langle B_{n}\left(D_{j, n} X_{j}, Y_{j}\right)+B_{n}\left(X_{j}, D_{j, n}\right), \alpha_{j}(K) X_{j}-\alpha_{j}(H) Y_{j}\right\rangle
$$

By (9)-(11), the first factors in these inner products satisfy (i). By Lemma 5.10, the second factors satisfy (ii) with $C_{n}$ independent of $n$.

Next we examine $\Psi_{n}=\Theta_{n}-\Phi_{n}$. Fix a pair of independent elements $\alpha$ and $\gamma$ in $a^{*}$ which arise as the real parts of roots of $a$ in $n$. Then $\alpha=c \alpha_{j}, \gamma=d \alpha_{k}$ for $c, d>0$ and $1 \leqslant j \neq k \leqslant m$. Call $X_{\alpha}^{1}$ (respectively, $Y_{\gamma}^{1}$ ) the component of $X$ 
(respectively, $Y$ ) in $\mathfrak{n}^{1} \cap \Sigma_{\beta} \mathfrak{n}_{\alpha, \beta}$ (respectively, $\mathfrak{n}^{1} \cap \Sigma_{\delta} \mathfrak{n}_{\gamma, \delta}$ ). Since $\left.E\right|_{\mathfrak{n}}$ is normal and $\left.T_{n}\right|_{n 1}=I$, it follows from (8) and (11) that $k_{n} X_{\alpha}^{1}=\alpha(K) X_{\alpha}^{1}, k_{n}\left(Y_{\gamma}^{1}\right)$ $=\gamma(K) Y_{\gamma}^{1}$. Moreover, $B_{n}\left(\mathfrak{n}_{j}, \mathfrak{n}_{k}\right)=B_{n}\left(\mathfrak{n}^{1} \cap \mathfrak{n}_{j}, \mathfrak{n}^{1} \cap \mathfrak{n}_{k}\right)$ and thus

$$
B_{n}\left(X_{\alpha}, k_{n} Y_{\gamma}\right)+B_{n}\left(k_{n} X_{\alpha}, Y_{\gamma}\right)=(\alpha(K)+\gamma(K)) B_{n}\left(X_{\alpha}^{1}, Y_{\gamma}^{1}\right)
$$

with a similar expression for $k_{n}$ replaced by $h_{n}$. Now let $l$ be the unique index such that $\alpha+\gamma=t_{l} \alpha_{l}$ for some $t_{l}>0$. Then $B_{n}\left(X_{\alpha}^{1}, Y_{\gamma}^{1}\right) \in \mathfrak{n}_{l}$ and hence is orthogonal to $\mathfrak{n}_{h}$ for all $h \neq l$. Consequently, the contribution to $\Psi_{n}$ involving $X_{\alpha}$ and $Y_{\gamma}$ is given by

$$
\begin{aligned}
\left\langle B_{n}\left(X_{\alpha}, k_{n} Y_{\gamma}\right)+B_{n}\left(k_{n} X_{\alpha}, Y_{\gamma}\right), X\right\rangle- & \left\langle B_{n}\left(X_{\alpha}, h_{n} Y_{\gamma}\right)+B_{n}\left(h_{n} X_{\alpha}, Y_{\gamma}\right), Y\right\rangle \\
& =t_{l}\left\langle B_{n}\left(X_{\alpha}^{1}, Y_{\gamma}^{1}\right), \alpha_{l}(K) X_{l}-\alpha_{l}(H) Y_{l}\right\rangle .
\end{aligned}
$$

Combining this with the contribution involving $X_{\gamma}$ and $Y_{\alpha}$, we obtain

$$
t_{l}\left\langle B_{n}\left(X_{\alpha}^{1}, Y_{\gamma}^{1}\right)+B_{n}\left(Y_{\gamma}^{1}, X_{\alpha}^{1}\right), \alpha_{l}(K) X_{l}-\alpha_{l}(H) Y_{l}\right\rangle
$$

As before, this expression is expressible in terms of operators satisfying (i) and (ii). Since $\Psi_{n}$ is simply the sum of such expressions over all independent $\alpha$ and $\gamma$, we have shown that $\left\langle R_{n}(X \wedge K-Y \wedge H), X \wedge Y\right\rangle$ is a finite sum of expressions satisfying (i) and (ii). Since the number of summands depends only on the number of roots and hence is independent of $n$, we conclude that the desired inequality (16) holds for $n$ sufficiently large. Q.E.D.

7.8. Corollary (see Heintze [4]). Let be a Lie algebra. Then carries a metric with strictly negative curvature if and only if 8 is an NC algebra with $\operatorname{dim} a=1$ and $\mathfrak{n}_{0}=\{0\}$, i.e. if and only if 8 is solvable, $\operatorname{codim}[8,8]=1$, and there exists $X \in \mathbb{8}$ such that the eigenvalues of $\operatorname{ad}_{\mathfrak{n}} X$ have strictly positive real parts.

Proof. The necessity follows directly from Lemma 5.10 and Theorem 7.6. The sufficiency is a direct consequence of the proof of Theorem 7.6 since for $n$ sufficiently large, $R_{n}$ is positive definite on $\left(\mathfrak{n}_{+} \wedge \mathfrak{n}_{+}+\Sigma_{1<j<m} \mathfrak{n}_{j} \wedge H_{j}\right)$ and this space coincides with $\Lambda^{2}$ when $\mathfrak{n}_{0}=\{0\}, \operatorname{dim} \mathfrak{a}=1$. Q.E.D.

7.9. Remarks. Corollaries 7.7 and 7.8 show that among manifolds with negative curvature, those with strictly negative curvature play approximately the role of rank 1 symmetric spaces among all noncompact symmetric spaces. Heintze's paper [4], in contrast to ours, shows that the study of the strictly negative case may be handled directly since most of the complexities of the general situation (e.g. deciding that $a$ is abelian, examining the subspace $L$ in $\Lambda^{\mathbf{2}} \mathbf{8}$, and establishing the decompositions $\mathfrak{n}_{\alpha, \beta}=\mathfrak{n}_{\alpha, \beta}^{0}+\mathfrak{n}_{\alpha, \beta}^{1}$ ) are then absent. The inner product on 8 constructed in Theorem 5.10 had the special property that all angles between real 
parts of roots (as elements of $a^{*}$ ) were acute. As was seen in Proposition 7.3 this is a necessary condition when $\mathfrak{n}$ is abelian. Our argument shows, roughly, that it is sufficient if $\mathfrak{n}$ is "almost" abelian. In general, however, this condition is by no means necessary. Indeed for $S$ an Iwasawa factor in a noncompact semisimple Lie group $G$, transportation of the metric from the symmetric space $G / K$ ( $K$ a maximal compact subgroup of $G$ ) to the Lie algebra of $S$ results essentially on $\boldsymbol{a}$ in the inner product induced by the Killing form of $\mathbf{g}$. As is well known, angles between simple roots relative to the Killing form are obtuse. The solvmanifolds with negative curvature actually constructed in the proof of Theorem 7.6 are thus clearly of a special nature which will be elucidated in Part II of this paper.

\section{Conclusion.}

8.1. Our approach throughout this paper has been to pass by an isometry from an arbitrary solvmanifold $M$ with negative curvature to a Lie group $S$ equipped with a left-invariant metric. We have shown that an arbitrary simply connected Lie group $S$ can arise in this way if and only if its Lie algebra 8 is an $N C$ algebra. Part II of our study will be devoted in large measure to a deeper look at the correspondence between $M$ and $S$. We note that at first glance, the correspondence seems to be rather weak. Indeed, it is not difficult to see that for a fixed $M$, there are, in general, infinitely many nonisomorphic Lie subgroups of $I(M)$ acting simply transitively on $M$ and consequently infinitely many structurally distinct choices for $S$. Conversely, for a fixed $N C$ algebra 8 , we have explicitly obtained in $\$ 7$ infinitely many distinct inner products on 8 relative to which $\&$ has negative curvature. It follows easily that there exist infinitely many nonisometric solvmanifolds $M$ all of which may be realized by various left-invariant metrics on the same topological group $S$. We now summarize the way in which the results of Part II will tighten up the correspondence.

In one direction, given $M$, one is interested in discovering the extent to which the Riemannian structure on $M$ determines the group structure of $S$. For this, it is of course necessary to examine the family $\mathfrak{F}(M)$ consisting of all Lie subgroups of $I(M)$ which act simply transitively on $M$. We will show that every element of $\mathfrak{F}(M)$ may be obtained from any fixed element by certain modifications and in the process determine which structural properties are common to all members of $\mathfrak{F}(M)$. In particular, we will see that there is a canonical isomorphism class within $\mathfrak{F}(M)$, i.e. for each $M$ we can use certain criteria to select a group $S(M) \in$ $\mathfrak{F}(M)$ in such a way that $M$ isometric to $M^{\prime}$ implies $S(M)$ isomorphic to $S\left(M^{\prime}\right)$. Among all isomorphism classes of $\mathfrak{Z}(M)$, the class of $S(M)$ will be shown to have optimal structural simplicity.

Conversely, suppose we have a fixed $N C$ algebra 8 . Let $M$ be a solvmanifold with negative curvature realized on the associated group $S$. We will see that 
knowledge of the root space structure of 8 allows one to read off the de Rham decomposition of $M$. Furthermore, for any inner product $\langle\cdot, \cdot\rangle$ on $\&$ relative to which 8 has negative curvature, the use of linear algebra alone allows one to explicitly construct $G=I_{0}(M)$ and there is a group-theoretic characterization of the Lie groups $G$ which can arise in this way.

8.2. We conclude by mentioning several related problems and lines of inquiry which we have not yet attempted to look into.

(i) Examine those manifolds which have a Kählerian (or, more generally, Hermitian) complex structure, are acted upon transitively by the group of holomorphic isometries, and have negative holomorphic curvature. In particular, determine which of these manifolds have negative Riemannian curvature. Recall that the holomorphic curvature of a Hermitian manifold $M$ at a point $p$ is just the Riemannian curvature restricted to those two-dimensional subspaces of $T_{p}(M)$ invariant under the complex structure map $J_{p}: T_{p}(M) \rightarrow T_{p}(M)$.

(ii) Discover the extent to which well-known results in harmonic analysis on noncompact symmetric spaces generalize to solvmanifolds with negative curvature. In particular, for any such manifold $M$, relate the geometry of $M$ to an explicit Plancherel formula for $L_{2}(M, \mu)$ where $\mu$ is the unique (up to scalar multiple) Borel measure on $M$ invariant under $I(M)$.

(iii) For $S$ as in 8.1, study a problem which strongly motivated one of the authors involving "laws of large numbers" for $S$-valued random variables. Thus, assign to $S$ a left-invariant metric with negative curvature and denote by $D(x, y)$ the associated Riemannian distance between two points $x$ and $y$ in $S$. Fix a Borel probability measure $\mu$ on $S$ and suppose there exists a number $\alpha \geqslant 1$ for which the integral

$$
J(\alpha, \mu, x)=\int_{S} D^{\alpha}(x, y) d \mu(y)
$$

is finite for some (and hence all) $x \in S$. Classical convexity properties of $D$ show that there exists a unique point $x(\alpha, \mu)$ in $S$ at which $J(\alpha, \mu, x)$ reaches an absolute minimum. What is the asymptotic behaviour of $x\left(\alpha, \mu^{* k}\right)$ as $k \rightarrow \infty$ ? Here, for $k$ a positive integer, $\mu^{* k}$ denotes the $k$-fold convolution power of $\mu$. When $\alpha=2$ and $S=\mathbf{R}^{n}, x(2, \mu)$ is simply the barycenter of $\mu$ and it is well known that $x\left(2, \mu^{* k}\right)=k \cdot x(2, \mu)$.

\section{REFERENCES}

1. R. Azencott and E. Wilson, Variétés homogènes à courbure négative, C. R. Acad. Sci. Paris Sér. A 278 (1974), 561-562.

2. A. Borel, Lectures on symmetric spaces, dittoed notes, Massachusetts Institute of Technology, 1958.

3. E. Cartan, Lecons sur la géométrie des espaces de Riemann, Gauthier-Villars, Paris, 1928. 
4. E. Heintze, On homogeneous manifolds of negative curvature (pre-publication manuscript).

5. S. Helgason, Differential geometry and symmetric spaces, Pure and Appl. Math., vol. 12, Academic Press, New York, 1962. MR 26 \#2986.

6. J. E. Humphreys, Introduction to Lie algebras and representation theory, Graduate Texts in Math., vol. 9, Springer-Verlag, New York and Berlin, 1972. MR 48 \#2197.

7. K. Iwasawa, On some types of topological subgroups, Ann. of Math. (2) 50 (1949), 507-558. MR 10, 679.

8. G. R. Jensen, Homogeneous Einstein spaces of dimension four, J. Differential Geometry 3 (1969), 309-349. MR 41 \#6100.

9. S. Kobayashi and K. Nomizu, Foundations of differential geometry. Vol. I, Interscience, New York, 1963. MR 27 \#2945.

10. A. Malcev, On the theory of Lie groups in the large, Mat. Sb. 16 (58) (1945), 163-190. MR 7, 115.

11. S. Myers and N. Steenrod, The group of isometries of a Riemannian manifold, Ann. of Math. 40 (1939), 400-416.

12. N. Wallach, Harmonic analysis on homogeneous spaces, Dekker, New York, 1973.

13. J. A. Wolf, Homogeneity and bounded isometries in manifolds of negative curvature, Illinois J. Math. 8 (1964), 14-18. MR 29 \#565.

DEPARTMENT OF MATHEMATICS, BRANDEIS UNIVERSITY, WALTHAM, MASSACHUSETTS 02154

Current address (R. Azencott): Unité Enseigment Recherche de Mathematiques, Université de Paris 7, Place Jussieu, Paris (5eme), France

Current address (E. N. Wilson): Department of Mathematics, Washington University, St. Louis, Missouri 63130 